\title{
Homogenization of oscillating boundaries and applications to thin films
}

\author{
Nadia Ansini and Andrea Braides \\ SISSA, via Beirut 4, 34013 Trieste, Italy \\ Email: ansini@sissa.it, braides@sissa.it
}

\section{Introduction}

The study carried on in this paper draws its motivation from the problem of the asymptotic description of nonlinearly elastic thin films with a fast-oscillating profile. The behaviour of such films is governed by an elastic energy, where two parameters intervene: a first parameter $\varepsilon$ represents the thickness of the thin film and a second one $\delta$ the scale of the oscillations. The analytic description of the elastic energy is given by a functional of the form

$$
E_{\varepsilon, \delta}(u)=\int_{\Omega(\varepsilon, \delta)} W(D u) d x,
$$

where the set $\Omega(\varepsilon, \delta)$ is of the form

$$
\Omega(\varepsilon, \delta)=\left\{x \in \mathbb{R}^{3}:\left|x_{3}\right|<\varepsilon f\left(\frac{x_{1}}{\delta}, \frac{x_{2}}{\delta}\right),\left(x_{1}, x_{2}\right) \in \omega\right\},
$$

with $f$ is a bounded 1-periodic function which parameterizes the boundary of the thin film, which then has periodicity $\delta$. It is convenient to scale these energies by a change of variables and consider the functionals

$$
E_{\varepsilon}^{\delta}(u)=\int_{\Omega(\delta)} W\left(D_{1} u, D_{2} u, \frac{1}{\varepsilon} D_{3} u\right) d x,
$$

where now

$$
\Omega(\delta)=\left\{x \in \mathbb{R}^{3}:\left|x_{3}\right|<f\left(\frac{x_{1}}{\delta}, \frac{x_{2}}{\delta}\right),\left(x_{1}, x_{2}\right) \in \omega\right\} .
$$

In this way we separate the effects of the two parameters $\varepsilon$ and $\delta$.

In a recent paper by Braides, Fonseca and Francfort [8] a general compactness result for functional of thin-film type has been proven which comprises energies of the form (1.3), showing that, with fixed $\delta=\delta(\varepsilon)$, upon possibly extracting a subsequence, the family $E_{\varepsilon}^{\delta(\varepsilon)}$ converges in the sense of De Giorgi's $\Gamma$-convergence 
as $\varepsilon \rightarrow 0$ to a 2-dimensional energy, which, if $\delta(\varepsilon) \rightarrow 0$ as $\varepsilon \rightarrow 0$, can be identified with a $2 d$-functional of the form

$$
E(u)=\int_{\omega} \widetilde{W}\left(D_{1} u, D_{2} u\right) d x .
$$

In many cases it is possible to describe $\widetilde{W}$ explicitly in terms of $W$ and $f$, and as a consequence to prove that no passage to a subsequence is necessary. When $f=C$ is constant (i.e., the profile of the thin film is flat, and hence there is no real dependence on $\delta$ ) the description of the energy density $\widetilde{W}$ has been given by Le Dret and Raoult [19] who proved that $\widetilde{W}=2 C Q_{2} \bar{W}$; here $Q_{2}$ denotes the operation of $2 d$-quasiconvexification, and $\bar{W}$ is obtained from $W$ by minimizing in the third component. An equivalent formula, of 'homogenization type', is given in [8] (see also [6]). If $\bar{W} \neq Q_{2} \bar{W}$ (i.e., $\bar{W}$ is not quasiconvex) then both formulas underline the formation of microstructures generated by the passage to the limit. When $f$ is not constant, then the function $W$ depends on the behaviour of $\delta$ with respect to $\varepsilon$. The case when $\delta=\varepsilon$ (or more in general when $\delta / \varepsilon$ converges to a constant) has been treated in [8], where it is shown that a homogenization type formula for $\widetilde{W}$ can be given. The same method can be used when $\delta>>\varepsilon$; in this case the recipe to obtain $\widetilde{W}$ is the following: first, keep $\delta$ fixed and apply the Le Dret and Raoult procedure, considering the thickness of the thin film as a parameter. The output of this procedure is a 2-dimensional energy of the form

$$
E^{\delta}(u)=\int_{\omega} 2 f\left(\frac{x_{1}}{\delta}, \frac{x_{2}}{\delta}\right) Q_{2} \bar{W}\left(D_{1} u, D_{2} u\right) d x .
$$

We can then let $\delta$ tend to 0 , and apply well-known homogenization procedures (see [6]) obtaining a limit functional, which turns out to be the desired one. In the case $\delta<<\varepsilon$ it is possible to make an ansatz in the same spirit, arguing that the limit $E$ can be obtained in the following two steps:

(1) (Homogenization of sets with oscillating boundaries) First consider $\varepsilon$ as fixed, and let $\delta \rightarrow 0$, to obtain a limit functional of the form

$$
E_{\varepsilon}(u)=\int_{\omega \times(-1,1)} W_{\mathrm{hom}}\left(x_{3}, D_{1} u, D_{2} u, \frac{1}{\varepsilon} D_{3} u\right) d x
$$

(we consider the normalized case $\sup f=1$ ).

Note that in this case an additional dependence on $x_{3}$ is introduced, which may underline a loss of coerciveness of the function $W_{\text {hom }}$ for certain values of $x_{3}$. The form of $W_{\text {hom }}$ will depend on $W$ and on the sublevel sets of $f$;

(2) (Thin film limit) Let $\varepsilon \rightarrow 0$ and generalize the method of [8] to noncoercive functionals. In this way we obtain a limit energy density

$$
\begin{aligned}
\bar{W}_{\text {hom }}(\bar{F})=\inf _{k \in \mathbb{N}} \inf \left\{\frac{1}{k^{2}} \int_{(0, k)^{2} \times(0,1)} W_{\text {hom }}\left(x_{3}, D u+(\bar{F}, 0)\right) d x:\right. \\
\left.u \in \mathrm{W}_{\text {loc }}^{1, p}\left((0,1)^{3} ; \mathbb{R}^{3}\right), u k \text {-periodic in }\left(x_{1}, x_{2}\right)\right\} .
\end{aligned}
$$


Note that the dependence on $x_{3}$ implies that the simpler method of [19] cannot be applied to this situation.

A partial result in this case has been obtained by Kohn and Vogelius 20] who dealt with linear operators.

The purpose of this work is twofold. First, we give a general theory for the homogenization of non-convex energies defined on sets with oscillating boundaries by generalizing the application of the direct methods of $\Gamma$-convergence to homogenization as described in [6]. We clarify and prove statement (1) above, by showing that the functionals $E_{\varepsilon}$ are defined on a 'degenerate Sobolev Space' that can be described by proving an auxiliary convex-homogenization result. The formula for $W_{\text {hom }}$ can be obtained by solving a possibly degenerate localized $3 d-$ homogenization problem. In the case of convex $W$ the determination of $W_{\text {hom }}(t, \bar{F})$ for fixed $t \in(-1,1)$ essentially amount to solving a $2 d$-homogenization problem with an energy which is coercive only on the set $E_{t}=\left\{\left(x_{1}, x_{2}\right) \in \mathbb{R}^{2}: f\left(x_{1}, x_{2}\right)>\right.$ $|t|\}$, while in the general non-convex case the problem defining $W_{\text {hom }}(t, \bar{F})$ is genuinely three dimensional. We state and prove these results in a general $n$ dimensional setting (for some related problems in the convex setting see e.g. (91).

The second goal of the paper is to prove that by following steps (1) and (2) above we indeed obtain the description of $\widetilde{W}$. Even though this is an intrinsically vectorial problem, and hence the 'natural' structural condition on $W$ is quasiconvexity, we have been able to prove this result only with the additional hypothesis that $W$ is convex. The technical point where this assumption is needed is the separation of scales argument, which assures that, essentially, homogenization comes first, followed by the thin film $3 d-2 d$ limit. In general problems where only quasiconvexity is assumed this point is usually proved by a compactness argument which uses some equi-integrability properties of gradients of optimal sequences for the homogenization derived from the growth conditions on the energy density (see e.g. Fonseca Müller Pedregal [17]; for the use of this argument in the framework of iterated homogenization see [6] Chapter 22; for an application to heterogeneous thin films with flat profile see Shu 23]). In the case of thin films with fast-oscillating profiles, this technique cannot be used since we have a control on the gradients of optimal sequences only on varying wildly oscillating domains. In the convex case though, optimal sequences for the homogenization can be obtained simply by scaling one single periodic function, and hence their gradients automatically enjoy equi-integrability properties. Note that this difficulty is similar to those encountered when dealing with higher-order theories of thin films. In that case the necessary compactness properties can be obtained by adding a small perturbation with higher-order derivatives (as in the paper by Bhattacharya and James [3]). We do not follow this type of argument since even a singular perturbation by higher-order gradients might interact with the homogenization process, as shown by Francfort and Müller [18]. More applications of $\Gamma$-convergence arguments to thin films theory can be found in [2, 7]. 


\section{Notation and Preliminaries}

In the sequel, $n, m \in \mathbb{N}$ with $n \geq 2, m \geq 1$. If $x \in \mathbb{R}^{n}$ then $x_{\alpha}=\left(x_{1}, \ldots, x_{n-1}\right) \in$ $\mathbb{R}^{n-1}$ is the vector of the first $n-1$ components of $x$, and $D_{\alpha}=\left(\frac{\partial}{\partial x_{1}}, \ldots, \frac{\partial}{\partial x_{n-1}}\right)$. If $\Omega$ is a open subset of $\mathbb{R}^{n}$ we denote by $\mathcal{A}(\Omega)$ the family of all open subsets of $\Omega$.

The notation $\mathbb{M}^{m \times n}$ stands for the space of $m \times n$ matrices. Given a matrix $F \in \mathbb{M}^{m \times n}$, and following the notation introduced in 19 , we write $F=\left(\bar{F} \mid F_{n}\right)$, where $F_{i}$ denotes the $i$-th column of $F, 1 \leq i \leq n$, and $\bar{F}=\left(F_{1}, \ldots, F_{n-1}\right) \in$ $\mathbb{M}^{m \times n-1}$ is the matrix of the first $n-1$ columns of $F \cdot \bar{F}$ denotes also $(\bar{F}, 0)$ when no confusion arises.

The characteristic function of a set $E \subset \mathbb{R}^{n}$ is denoted by $\chi_{E}$, and the $N$-dimensional Lebesgue measure in $\mathbb{R}^{N}$ is designated as $\mathcal{L}^{N}$. We use standard notation for Lebesgue and Sobolev spaces. The letter $c$ will stand for an arbitrary fixed strictly-positive constant.

We recall the definition of De Giorgi's $\Gamma$-convergence in $\mathrm{L}^{p}$ spaces, $1 \leq p<$ $+\infty$. Given a family of functionals $J_{j}: \mathrm{L}^{p}\left(\Omega ; \mathbb{R}^{m}\right) \rightarrow[0,+\infty), j \in \mathbb{N}$, for $u \in$ $\mathrm{L}^{p}\left(\Omega ; \mathbb{R}^{m}\right)$ we define

$$
\Gamma-\liminf _{j \rightarrow+\infty} J_{j}(u)=\inf \left\{\liminf _{j \rightarrow+\infty} J_{j}\left(u_{j}\right): u_{j} \rightarrow u \text { in } \mathrm{L}^{p}\left(\Omega ; \mathbb{R}^{m}\right)\right\},
$$

and

$$
\Gamma-\limsup _{j \rightarrow+\infty} J_{j}(u)=\inf \left\{\limsup _{j \rightarrow+\infty} J_{j}\left(u_{j}\right): u_{j} \rightarrow u \text { in } \mathrm{L}^{p}\left(\Omega ; \mathbb{R}^{m}\right)\right\} .
$$

If these two quantities coincide then their common value is called the $\Gamma$-limit of the sequence $\left(J_{j}\right)$ at $u$, and is denoted by $\Gamma-\lim _{j \rightarrow+\infty} J_{j}(u)$. It is easy to check that $l=\Gamma-\lim _{j \rightarrow+\infty} J_{j}(u)$ if and only if

(a) for every sequence $\left(u_{j}\right)$ converging to $u$ in $\mathrm{L}^{p}\left(\Omega ; \mathbb{R}^{m}\right)$ we have

$$
l \leq \liminf _{j \rightarrow+\infty} J_{j}\left(u_{j}\right)
$$

(b) there exists a sequence $\left(u_{j}\right)$ converging to $u$ in $\mathrm{L}^{p}\left(\Omega ; \mathbb{R}^{m}\right)$ such that

$$
l \geq \limsup _{j \rightarrow+\infty} J_{j}\left(u_{j}\right) .
$$

We say that $\left(J_{\varepsilon}\right) \Gamma$-converges to $l$ at $u$ as $\varepsilon \rightarrow 0$ if for every sequence of positive numbers $\left(\varepsilon_{j}\right)$ converging to 0 there exists a subsequence $\left(\varepsilon_{j_{k}}\right)$ for which

$$
l=\Gamma-\lim _{k \rightarrow+\infty} J_{\varepsilon_{j_{k}}}(u) .
$$

We recall that the $\Gamma$-upper and lower limits defined above are $\mathrm{L}^{p}$-lower semicontinuous functions.

For a comprehensive study of $\Gamma$-convergence we refer to the book of Dal Maso [13] (for a simplified introduction see [5]), while a detailed analysis of some of its applications to homogenization theory can be found in [6]. 


\section{The direct method of $\Gamma$-convergence}

In the sequel we will repeatedly apply some variants of the so-called direct method of $\Gamma$-convergence to homogenization problems, which consists in combining localization and integral representation procedures to obtain compactness theorem for classes of integral functional. This method in the version which follows is explained in detail in the book by Braides and Defranceschi [6] (see also Dal Maso [13] and Buttazzo [10]).

Let $\Omega$ be a bounded subset of $\mathbb{R}^{n}$, let $p>1$ and let $F_{\varepsilon}: \mathrm{L}^{p}\left(\Omega ; \mathbb{R}^{m}\right) \times \mathcal{A}(\Omega) \rightarrow$ $[0,+\infty]$ be a family of functionals of the form

$$
F_{\varepsilon}(u, U)= \begin{cases}\int_{U} f_{\varepsilon}(x, D u) d x & \text { if } u \in X_{\varepsilon}(U) \\ +\infty & \text { otherwise }\end{cases}
$$

for suitable function spaces $X_{\varepsilon}(U)$ and $f_{\varepsilon}: \mathbb{R}^{n} \times \mathbb{M}^{m \times n} \rightarrow[0,+\infty)$ Borel functions. Suppose that there exist Borel functions $g_{\varepsilon}: \mathbb{R}^{n} \times \mathbb{R} \rightarrow[0,+\infty)$, convex and even in the second variable, with

$$
\begin{aligned}
g_{\varepsilon}(x,|F|) & \leq f_{\varepsilon}(x, F) \leq C\left(1+g_{\varepsilon}(x,|F|)\right) \leq C\left(1+|F|^{p}\right), \\
g_{\varepsilon}(x, 2 t) & \leq C\left(1+g_{\varepsilon}(x, t)\right)
\end{aligned}
$$

for all $F \in \mathbb{M}^{m \times n}, x \in \Omega$ and $t \in \mathbb{R}$. Growth conditions (3.2) and (3.3) are designed to include functions of the type $a_{\varepsilon}(x)|F|^{p}$ with the only assumption $a_{\varepsilon} \geq 0$, thus allowing for zones where $a_{\varepsilon}=0$. In the next section $a_{\varepsilon}$ will be the characteristic function of a set with fast-oscillating boundary. Note that a general theory for functions satisfying

$$
0 \leq f_{\varepsilon}(x, F) \leq C\left(1+|F|^{p}\right)
$$

only has not be developed yet. The aim of the direct method of $\Gamma$-convergence is to prove a compactness result for the family $\left(F_{\varepsilon}\right)$, giving a representation of the limit, and, possibly, complete the description in terms of 'homogenization formulas'.

Step 1 With fixed $\left(\varepsilon_{j}\right)$ extract a subsequence (not relabeled) such that $F_{\varepsilon}(\cdot, U) \Gamma$-converges to a functional $F_{0}(\cdot, U)$ for all $U$ in a dense family of open sets $\mathcal{U}$ (see [6] Proposition 7.9);

Step 2 Thanks to (3.2) and (3.3), prove that $F_{0}(u, \cdot)$ is the restriction of a finite Borel measure to $\mathcal{U}$ for all $u \in \mathrm{W}^{1, p}\left(\Omega ; \mathbb{R}^{m}\right)$, so that by inner regularity we indeed have that $F_{\varepsilon}(\cdot, U) \Gamma$-converges to a functional $F_{0}(\cdot, U)$ on $\mathrm{W}^{1, p}\left(\Omega ; \mathbb{R}^{m}\right)$ for all $U \in \mathcal{A}(\Omega)$. In this step is crucial the so-called fundamental $\mathrm{L}^{p}$-estimate: for all $U, Y, Z \in \mathcal{A}(\Omega)$ with $Y \subset \subset U$, and for all $\sigma>0$, there exists $M>0$ such that for all $u, v \in \mathrm{W}^{1, p}\left(\Omega ; \mathbb{R}^{m}\right)$ one may find a cut-off function $\varphi \in C_{0}^{\infty}(U ;[0,1]), \varphi=1$ in $Y$, such that

$$
\begin{aligned}
F_{\varepsilon}(\varphi u+(1-\varphi) v, Y \cup Z) \leq & (1+\sigma)\left(F_{\varepsilon}(u, U)+F_{\varepsilon}(v, Z)\right) \\
& +M \int_{(U \cap Z) \backslash Y}|u-v|^{p} d x+\sigma .
\end{aligned}
$$


Moreover, by again using the fundamental $\mathrm{L}^{p}$-estimate it can be proven that if $u \in \mathrm{W}^{1, p}\left(\Omega ; \mathbb{R}^{m}\right) \cap X_{\varepsilon}(U)$ for all $\varepsilon$ and $F_{0}(u, U)<+\infty$ then there exist a sequence $u_{\varepsilon} \in X_{\varepsilon}(U)$ such that

$$
\lim _{\varepsilon \rightarrow 0} F_{\varepsilon}\left(u_{\varepsilon}, U\right)=F_{0}(u, U)
$$

and $u_{\varepsilon}=u$ on a neighbourhood of $\partial U$ (see [6] Chapter 11);

Step 3 By the locality and semicontinuity properties of $\Gamma$-limits and by Step 2 we can find a function $\varphi: \Omega \times \mathbb{M}^{m \times n} \rightarrow[0,+\infty)$ such that $0 \leq \varphi(x, F) \leq$ $C\left(1+|F|^{p}\right)$ and $F_{0}(u, U)=F_{\varphi}(u, U)$ for all $u \in \mathrm{W}^{1, p}\left(\Omega ; \mathbb{R}^{m}\right)$ and $U \in \mathcal{A}(\Omega)$, where

$$
F_{\varphi}(u)=\int_{\Omega} \varphi(x, D u) d x .
$$

In the proof of this step a crucial point is the passage from the identity $F_{0}(u)=$ $F_{\varphi}(u)$ when $u$ is piecewise affine to a general $u$ by the continuity of $F_{\varphi}$ with respect to a convergence in which piecewise-affine functions are dense (e.g. the

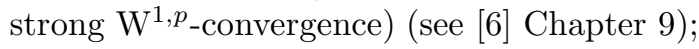

Step 4 If $f_{\varepsilon}(x, F)=f\left(\frac{x}{\varepsilon}, F\right)$ with $f 1$-periodic in the first variable then by the periodicity of $f$ we deduce that $\varphi=\varphi(F)$ (see [6] Proposition 14.3);

Step 5 If $g_{\varepsilon}(x, F)=g\left(\frac{x}{\varepsilon}, F\right)$ with $g$ 1-periodic in the first variable then we consider the auxiliary functionals

$$
G_{\varepsilon}(u, U)= \begin{cases}\int_{U} g_{\varepsilon}(x, D u) d x & \text { if } u \in X_{\varepsilon}(\Omega) \\ +\infty & \text { otherwise. }\end{cases}
$$

By Step $1-4$ we can assume that a function $\psi$ exists such that $G_{\varepsilon}(\cdot, U) \Gamma$-converges to the functional $F_{\psi}(\cdot, U)$ on $\mathrm{W}^{1, p}\left(\Omega ; \mathbb{R}^{m}\right)$ for all $U \in \mathcal{A}(\Omega)$;

Step 6 Note that $\psi$ is convex. By an argument of approximation by convolution prove that indeed the functional $G_{\varepsilon}(\cdot, U) \Gamma$-converges to the functional $F_{\psi}(\cdot, U)$ on $\mathrm{W}^{1,1}\left(\Omega ; \mathbb{R}^{m}\right)$ for all $U \in \mathcal{A}(\Omega)$. Define the 'domain' of $F_{\psi}(\cdot, \Omega)$ : $\mathrm{W}^{1, \psi}\left(\Omega ; \mathbb{R}^{m}\right)=\left\{u \in \mathrm{W}^{1,1}\left(\Omega ; \mathbb{R}^{m}\right): F_{\psi}(u, \Omega)<+\infty\right\}$ (see [6] Theorem 14.8);

Step 7 Repeat Step 2 and 3 substituting the space $\mathrm{W}^{1, p}\left(\Omega ; \mathbb{R}^{m}\right)$ by the space $\mathrm{W}^{1, \psi}\left(\Omega ; \mathbb{R}^{m}\right)$ thus obtaining the representation $F_{0}=F_{\varphi}$ on $\mathrm{W}^{1,1}\left(\Omega ; \mathbb{R}^{m}\right) ;$

Step 8 Deduce that $\varphi$ and $\psi$ do not depend on $\left(\varepsilon_{j}\right)$ by proving a homogenization formula (see [6] Proposition 21.12);

Step 9 Finally, the representation of $F_{0}$ on the whole $\mathrm{L}^{p}\left(\Omega ; \mathbb{R}^{m}\right)$, and not only on $\mathrm{W}^{1,1}\left(\Omega ; \mathbb{R}^{m}\right)$, can be obtained in some cases by a more accurate study of the properties of $\varphi$.

We will have to modify Steps 1-9 above as to cover the case when the domain of the limit is a 'degenerate Sobolev Space'. In particular, since the function $\psi$ obtained as in Step 5 will be degenerate, a suitable weighted Sobolev Space will have to be defined, which takes the place of $\mathrm{W}^{1,1}\left(\Omega ; \mathbb{R}^{m}\right)$ in Step 6 above. Moreover, we will have to deal with the fact that our functions $f_{\varepsilon}, g_{\varepsilon}$ may be periodic only in some variables, so that Step 8 will be harder to verify. We will include all the 
details of the reasonings which do not fall directly in this scheme, while we will feel free to refer to [6] for those procedures which have become customary.

It is worth mentioning that in some cases the arguments outlined above can be simplified by using some techniques (as blow-up arguments or the theory of Young measures) that avoid to use the complex localization procedure. As our problem is concerned those methods seem harder to apply since the energies we consider are coercive only on wildly oscillating sets.

\section{Homogenization of media with oscillating pro- file}

Let $f: \mathbb{R}^{n-1} \mapsto[0,1]$ be a 1 -periodic lower semicontinuous function and $0 \leq$ $\min f \leq \sup f=1$, let $W: \mathbb{R}^{n-1} \times \mathbb{M}^{m \times n} \mapsto[0,+\infty)$ be a Borel function 1periodic in the first variable satisfying

$$
\gamma|F|^{p} \leq W\left(x_{\alpha}, F\right) \leq \beta\left(1+|F|^{p}\right)
$$

for all $x_{\alpha} \in \mathbb{R}^{n-1}$ and $F \in \mathbb{M}^{m \times n}$, for some $1<p<+\infty, 0<\gamma \leq \beta$. The set $\omega$ will be a fixed bounded open subset of $\mathbb{R}^{n-1}$ with Lipschitz boundary and $\Omega=\omega \times(-1,1)$.

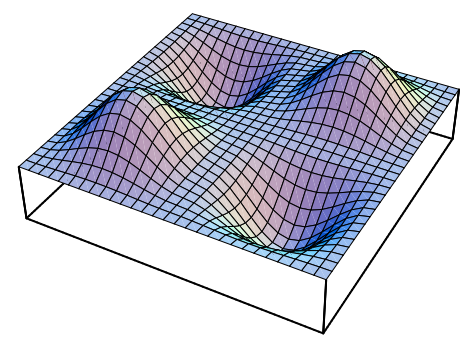

Figure 1: the graph of a typical $f$ in the unit cell

In this section we compute the $\Gamma$-limit of functionals of the form

$$
J_{\varepsilon}(u)= \begin{cases}\int_{\Omega_{\varepsilon}} W\left(\frac{x_{\alpha}}{\varepsilon}, D u\right) d x & \text { if } u_{\mid \Omega_{\varepsilon}} \in \mathrm{W}^{1, p}\left(\Omega_{\varepsilon} ; \mathbb{R}^{m}\right) \\ +\infty & \text { otherwise }\end{cases}
$$

where

$$
\Omega_{\varepsilon}=\left\{x \in \Omega:\left|x_{n}\right|<f\left(x_{\alpha} / \varepsilon\right)\right\} .
$$




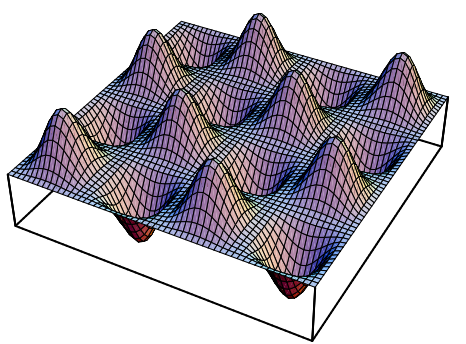

Figure 2: the upper profile of $\Omega_{\varepsilon}$ with $f$ as in Figure 1

The $\Gamma$-limit theorem will be stated and proved at the end of the section after some preliminary results, which are needed to define the domain of the $\Gamma$-limit and to explain the homogenization formula.

In orded to apply the method described in the previous section we introduce the localized version of the functionals $J_{\varepsilon}$ : for all $U$ open subset of $\Omega$ we define

$$
J_{\varepsilon}(u, U)= \begin{cases}\int_{\Omega_{\varepsilon} \cap U} W\left(\frac{x_{\alpha}}{\varepsilon}, D u\right) d x & \text { if } u_{\mid \Omega_{\varepsilon} \cap U} \in \mathrm{W}^{1, p}\left(\Omega_{\varepsilon} \cap U ; \mathbb{R}^{m}\right) \\ +\infty & \text { otherwise, }\end{cases}
$$

so that $J_{\varepsilon}(u)=J_{\varepsilon}(u, \Omega)$.

The first proposition contains the analog of Steps 1-4 of the direct method of $\Gamma$-convergence as outlined in the previous section.

Proposition 4.1 From every sequence $\left(\varepsilon_{j}\right)$ of positive numbers converging to 0 we can extract a subsequence (not relabeled) such that the $\Gamma$-limit

$$
J_{0}(u, U)=\Gamma-\lim _{j \rightarrow+\infty} J_{\varepsilon_{j}}(u, U)
$$

exists for all $u \in \mathrm{W}^{1, p}\left(\Omega ; \mathbb{R}^{m}\right)$ and $U$ open subsets of $\Omega$. Moreover, there exists a Carathéodory function $\varphi:(-1,1) \times \mathbb{M}^{m \times n} \rightarrow[0,+\infty)$ such that

$$
J_{0}(u, U)=\int_{U} \varphi\left(x_{n}, D u\right) d x
$$

for all $u \in \mathrm{W}^{1, p}\left(\Omega ; \mathbb{R}^{m}\right)$.

Proof. The functional $J_{\varepsilon}$ can be rewritten on $X_{\varepsilon}(U)=\left\{u \in \mathrm{L}^{p}\left(\Omega ; \mathbb{R}^{m}\right)\right.$ : $\left.u_{\mid \Omega_{\varepsilon} \cap U} \in \mathrm{W}^{1, p}\left(\Omega_{\varepsilon} \cap U ; \mathbb{R}^{m}\right)\right\}$ as

$$
J_{\varepsilon}(u, U)=\int_{U} \chi_{\Omega_{\varepsilon}}(x) W\left(\frac{x_{\alpha}}{\varepsilon}, D u\right) d x .
$$


We can then apply Steps 1-3 of Section 3 (see [6] Example 11.4 for the proof of the $\mathrm{L}^{p}$-fundamental estimate). Finally, a translation argument in the $x_{\alpha}$-plane (completely analogous, e.g., to the one in the proof of [6] Proposition 14.3) shows that

$$
\int_{B_{\rho}\left(x_{\alpha}\right) \times(z-\eta, z+\eta)} \varphi(y, F) d y=\int_{B_{\rho}\left(x_{\alpha}^{\prime}\right) \times(z-\eta, z+\eta)} \varphi(y, F) d y
$$

for all $\rho, \eta>0, x_{\alpha}, x_{\alpha}^{\prime}, z$ such that

$$
\left(B_{\rho}\left(x_{\alpha}\right) \times(z-\eta, z+\eta)\right) \cup\left(B_{\rho}\left(x_{\alpha}^{\prime}\right) \times(z-\eta, z+\eta)\right) \subset \Omega .
$$

We then easily deduce that $\varphi(x, F)=\varphi\left(x_{n}, F\right)$.

We will complete the proof of the homogenization theorem by characterizing the function $\varphi$ above (showing in particular that it does not depend on the sequence $\left.\left(\varepsilon_{j}\right)\right)$, proving the existence of the $\Gamma$-limit $J_{0}$ on the whole $L^{p}\left(\Omega ; \mathbb{R}^{m}\right)$ and showing that the integral representation in the previous proposition holds on the whole domain of $J_{0}$. In order to get to this result, we will have to define a number of auxiliary energies; here we streamline the organization of the rest of the section. First, in Section 4.1 we consider the case when $W(F)=\|F\|^{p}$. We will denote by $\psi$ the function given by Proposition 4.1 corresponding to this particular choice of $W$. For fixed $t$ the function $\psi(t, \cdot)$ is easily characterized by solving a $(n-1)$-dimensional (possibly, non coercive) homogenization problem. It is possible then to define the 'degenerate Sobolev Space' $\mathrm{W}_{\psi}^{1, p}\left(\Omega ; \mathbb{R}^{m}\right)$ of functions such that $\int_{\Omega} \psi\left(x_{n}, D u\right) d x<+\infty$, which turns out to be the domain of the $\Gamma$-limit when $W(F)=\|F\|^{p}$, and hence also in the general case by (4.1). In Section 4.2, in order to describe the function $\varphi$ in the general case, with fixed $t$ we consider the case when we replace the function $f$ by the characteristic function of $E_{t}=\left\{x_{\alpha}: f\left(x_{\alpha}\right)>|t|\right\}$ (i.e., we deal with cylindrical domains). The function $\varphi(t, \cdot)$ will eventually be given by the energy density of the corresponding $\Gamma$-limit. Finally, in Section 4.3 we are able to consider general $W$ and $f$ and obtain the oscillating-boundary homogenization Theorem 4.15 as the consequence of the previous sections.

\subsection{An auxiliary problem. Definition of the limit domain}

In general, the limit functional $J_{0}$ exists and is finite also outside $\mathrm{W}^{1, p}\left(\Omega ; \mathbb{R}^{m}\right)$. We first deal with the case of $J_{0}$ corresponding to

$$
W(x, F)=\|F\|^{p} \text {, where }\|F\|^{p}=\sum_{j=1}^{n}\left|F_{j}\right|^{p} .
$$

By a careful description of the domain of the corresponding $\Gamma$-limit we will identify the domain of $J_{0}$ as a suitable 'degenerate Sobolev Space' (see Definition 
4.5) which, in view of the growth condition (4.1), will also be the domain of $J_{0}$ corresponding to energy densities other than (4.5).

We recall a preliminary result.

Theorem 4.2 Let $E$ be a 1-periodic set in $\mathbb{R}^{N}$; i.e., such that $\chi_{E}$ is a 1-periodic function, and let

$$
J_{\varepsilon}^{E}(v, U)= \begin{cases}\int_{U \cap \varepsilon E}\|D v\|^{p} d x & \text { if } v_{\mid U \cap \varepsilon E} \in \mathrm{W}^{1, p}\left(U \cap \varepsilon E ; \mathbb{R}^{m}\right) \\ +\infty & \text { otherwise. }\end{cases}
$$

Then the $\Gamma$-limit

$$
J_{\text {hom }}^{E}(v, U)=\Gamma-\lim _{\varepsilon \rightarrow 0} J_{\varepsilon}^{E}(v, U)
$$

exists for all $U$ bounded open subsets of $\mathbb{R}^{N}$ and $v \in \mathrm{W}^{1, p}\left(U ; \mathbb{R}^{m}\right)$. Moreover, we have

$$
J_{\mathrm{hom}}^{E}(v, U)=\int_{U} \varphi_{\mathrm{hom}}^{E}(D v) d x
$$

for all $u \in \mathrm{W}^{1, p}\left(U ; \mathbb{R}^{m}\right)$, where $\varphi_{\mathrm{hom}}^{E}$ is a positively homogeneous function of degree $p$, satisfying the formula

$$
\varphi_{\text {hom }}^{E}(F)=\inf \left\{\int_{E \cap(0,1)^{N}}\|D v+F\|^{p} d x: v \in \mathrm{W}_{\text {loc }}^{1, p}\left(E ; \mathbb{R}^{m}\right), 1 \text {-periodic }\right\} .
$$

PROOF. This theorem is a particular case of [6] Theorem 14.8, the positive homogeneity of $\varphi_{\text {hom }}^{E}$ easily following from its definition.

For all $t \in(-1,1)$ we define

$$
\varphi_{\#}(t, \bar{F})=\varphi_{\text {hom }}^{E_{t}}(\bar{F}),
$$

the latter function being that given by the previous theorem, with $N=n-1$ and $E=E_{t}=\left\{x_{\alpha}: f\left(x_{\alpha}\right)>|t|\right\}$. We define also

$$
\psi(t, F)=\varphi_{\#}(t, \bar{F})+\mathcal{L}_{n-1}\left(E_{t} \cap(0,1)^{n-1}\right)\left|F_{n}\right|^{p} .
$$

Theorem 4.3 If $W=\|F\|^{p}$ and $\varphi$ is given by Proposition 1.1 then we have

$$
\varphi(t, F)=\psi(t, F) .
$$

In particular $\varphi$ does not depend on $\left(\varepsilon_{j}\right)$.

Proof. Let $(x, F)$ be such that $x_{n}$ is a Lebesgue point for $\varphi(\cdot, F)$. Then

$$
\begin{aligned}
\varphi\left(x_{n}, F\right) & =\lim _{\rho \rightarrow 0^{+}} f_{B_{\rho}\left(x_{\alpha}\right) \times\left(x_{n}-\rho, x_{n}\right)} \varphi\left(y_{n}, F\right) d y \\
& =\lim _{\rho \rightarrow 0^{+}} \frac{J_{0}\left(F y, B_{\rho}\left(x_{\alpha}\right) \times\left(x_{n}-\rho, x_{n}\right)\right)}{\left|B_{\rho}\left(x_{\alpha}\right) \times\left(x_{n}-\rho, x_{n}\right)\right|} .
\end{aligned}
$$


We consider the case $x_{n}>0$ only, the case $x_{n}<0$ being dealt with using a symmetric argument. Note that for $0<t<s<1$ we have $E_{s} \subseteq E_{t}$. Let $u_{j} \rightarrow 0$ with $u_{j} \in \mathrm{W}_{0}^{1, p}\left(B_{\rho}\left(x_{\alpha}\right) \times\left(x_{n}-\rho, x_{n}\right) \cap \Omega_{\varepsilon_{j}}\right)$ be such that

$$
J_{0}\left(F y, B_{\rho}\left(x_{\alpha}\right) \times\left(x_{n}-\rho, x_{n}\right)\right)=\lim _{j \rightarrow+\infty} J_{\varepsilon_{j}}\left(F y+u_{j}, B_{\rho}\left(x_{\alpha}\right) \times\left(x_{n}-\rho, x_{n}\right)\right) .
$$

Then,

$$
\begin{aligned}
& J_{\varepsilon_{j}}\left(F y+u_{j}, B_{\rho}\left(x_{\alpha}\right) \times\left(x_{n}-\rho, x_{n}\right)\right) \\
= & \int_{x_{n}-\rho}^{x_{n}} \int_{B_{\rho}\left(x_{\alpha}\right)} \chi_{E_{y_{n}}}\left(\frac{y_{\alpha}}{\varepsilon_{j}}\right)\left\|\bar{F}+D_{\alpha} u_{j}\right\|^{p} d y_{\alpha} d y_{n} \\
& +\int_{B_{\rho}\left(x_{\alpha}\right)} \int_{x_{n}-\rho}^{x_{n}} \chi_{E_{y_{n}}}\left(\frac{y_{\alpha}}{\varepsilon_{j}}\right)\left|F_{n}+D_{n} u_{j}\right|^{p} d y_{n} d y_{\alpha} \\
\geq & \int_{x_{n}-\rho}^{x_{n}} \int_{B_{\rho}\left(x_{\alpha}\right)} \chi_{E_{x_{n}}}\left(\frac{y_{\alpha}}{\varepsilon_{j}}\right)\left\|\bar{F}+D_{\alpha} u_{j}\right\|^{p} d y_{\alpha} d y_{n} \\
& +\rho \int_{B_{\rho}\left(x_{\alpha}\right)} \chi_{E_{x_{n}}}\left(\frac{y_{\alpha}}{\varepsilon_{j}}\right)\left|F_{n}\right|^{p} d y_{\alpha}
\end{aligned}
$$

by Jensen's inequality. By using the lower limit inequality for the $\Gamma$-convergence in Theorem 4.2 with $E=E_{x_{n}}$, and by an application of Fatou's Lemma, we get

$$
\begin{gathered}
J_{0}\left(F y, B_{\rho}\left(x_{\alpha}\right) \times\left(x_{n}-\rho, x_{n}\right)\right) \geq \rho \int_{B_{\rho}\left(x_{\alpha}\right)} \varphi_{\#}\left(x_{n}, \bar{F}\right) d y_{\alpha} \\
+\rho \mathcal{L}_{n-1}\left(B_{\rho}\left(x_{\alpha}\right)\right)\left|F_{n}\right|^{p} \mathcal{L}_{n-1}\left(E_{x_{n}} \cap(0,1)^{n-1}\right) .
\end{gathered}
$$

Letting $\rho \rightarrow 0^{+}$we obtain then by (4.8)

$$
\varphi\left(x_{n}, F\right) \geq \varphi_{\#}\left(x_{n}, \bar{F}\right)+\mathcal{L}_{n-1}\left(E_{x_{n}} \cap(0,1)^{n-1}\right)\left|F_{n}\right|^{p} .
$$

Vice versa, let $v_{j} \rightarrow 0$ be such that $\bar{F} y_{\alpha}+v_{j}\left(y_{\alpha}\right)$ is a recovery sequence for $J_{\text {hom }}^{E_{x_{n}}}\left(\bar{F} y_{\alpha}, B_{\rho}\left(x_{\alpha}\right)\right)$ along the sequence $\left(\varepsilon_{j}\right)$, and set

$$
u_{j}(y)=F y+\left(v_{j}\left(y_{\alpha}\right), 0\right)=\left(\bar{F} y_{\alpha}+v_{j}\left(y_{\alpha}\right), F_{n} y_{n}\right) .
$$

We then have

$$
\begin{aligned}
& \int_{B_{\rho}\left(x_{\alpha}\right) \times\left(x_{n}, x_{n}+\rho\right)} \varphi\left(y_{n}, A\right) d y \\
& \quad \leq \liminf _{j \rightarrow+\infty} J_{\varepsilon_{j}}\left(u_{j}, B_{\rho}\left(x_{\alpha}\right) \times\left(x_{n}, x_{n}+\rho\right)\right) \\
& \quad \leq \liminf _{j \rightarrow+\infty} \int_{B_{\rho}\left(x_{\alpha}\right) \times\left(x_{n}, x_{n}+\rho\right)} \chi_{E_{x_{n}}}\left(\frac{y_{\alpha}}{\varepsilon_{j}}\right)\left\|D u_{j}\right\|^{p} d y \\
& \quad=\lim _{j \rightarrow+\infty} \rho \int_{B_{\rho}\left(x_{\alpha}\right)} \chi_{E_{x_{n}}}\left(\frac{y_{\alpha}}{\varepsilon_{j}}\right)\left(\left\|\bar{F}+D_{\alpha} v_{j}\right\|^{p}+\left|F_{n}\right|^{p}\right) d y_{\alpha}
\end{aligned}
$$




$$
\begin{aligned}
=\rho \int_{B_{\rho}\left(x_{\alpha}\right)} \varphi_{\#}\left(x_{n}, \bar{F}\right) d y_{\alpha} \\
\quad+\rho \mathcal{L}_{n-1}\left(B_{\rho}\left(x_{\alpha}\right)\right)\left|F_{n}\right|^{p} \mathcal{L}_{n-1}\left(E_{x_{n}} \cap(0,1)^{n-1},\right.
\end{aligned}
$$

which gives the missing inequality by (4.8).

Remark 4.4 With fixed $t$, we define the 'kernel' of $\varphi_{\#}(t, \cdot)$ as

$$
\operatorname{Ker} \varphi_{\#}=\left\{\varphi_{\#}(t, \cdot)=0\right\} .
$$

Then $\operatorname{Ker} \varphi_{\#}$ is a linear space and its dimension is a multiple integer of $m$; i.e.,

$$
\operatorname{dim} \operatorname{Ker} \varphi_{\#}=k m \quad \text { for some } \quad k=0, \ldots, n-1
$$

and there exist $\xi_{k+1}, \ldots, \xi_{n-1} \in \mathbb{R}^{n-1}$ such that

$$
\bar{F}=\left(\begin{array}{c}
F^{1} \\
\vdots \\
F^{m}
\end{array}\right) \in \operatorname{Ker} \varphi_{\#} \quad \Leftrightarrow \quad \bar{F} \xi_{i}=0
$$

for each $i=k+1, \ldots, n-1$. (Note that $k$ depends on $t$ fixed and $F^{i}$ denotes the $i$-th row of $\bar{F}, 1 \leq i \leq m)$.

In fact, since $\bar{F} \mapsto \varphi_{\#}(t, \bar{F})$ is positively homogeneous of degree $p$, convex and even, $\operatorname{Ker} \varphi_{\#}$ is a linear space and satisfies the following properties: if $\bar{F} \in \operatorname{Ker} \varphi_{\#}$ then

(i) for each $\left(s_{1}, \ldots, s_{m}\right) \in \mathbb{R}^{m}$

$$
\left(\begin{array}{c}
s_{1} F^{1} \\
\vdots \\
s_{m} F^{m}
\end{array}\right) \in \operatorname{Ker} \varphi_{\#}
$$

(ii) $P \bar{F} \in \operatorname{Ker} \varphi_{\#}$ for each permutation matrix $P \in \mathbb{M}^{m \times m}$.

Properties (i) and (ii) imply that if we fix $F^{1}$ we can construct $m$ matrices linearly independent

$$
\left(\begin{array}{c}
F^{1} \\
0 \\
\vdots \\
0
\end{array}\right),\left(\begin{array}{c}
0 \\
F^{1} \\
\vdots \\
0
\end{array}\right), \cdots,\left(\begin{array}{c}
0 \\
0 \\
\vdots \\
F^{1}
\end{array}\right) \in \operatorname{Ker} \varphi_{\#}
$$

which span a subspace $\left\langle F^{1}\right\rangle$ of $\operatorname{Ker} \varphi_{\#}$ of dimension $m$.

Now, if $\left\langle F^{1}\right\rangle \neq \operatorname{Ker} \varphi_{\#}$, we can single out a non-zero matrix in $\operatorname{Ker} \varphi_{\#}$ orthogonal to $\left\langle F^{1}\right\rangle$, and, by using the same argument as above taking its first row 
vector, find other $m$ matrices which, together with the matrices constructed before, form a linearly independent family.

By proceeding in this way, we end up with $\eta_{1}, \ldots, \eta_{k} \in \mathbb{R}^{n-1}$ such that for all $A \in \operatorname{Ker} \varphi_{\#}$

$$
A^{i}=\sum_{j=1}^{k} s_{i j} \eta_{j} \quad i=1, \ldots, m
$$

with $s_{i j} \in \mathbb{R}$, which means that the $\operatorname{dim} \operatorname{Ker} \varphi_{\#}=k m$ for some $k \in\{1, \ldots, n-1\}$.

The orthogonal subspace to $\left\langle\eta_{1}, \ldots, \eta_{k}\right\rangle$ is a vector subspace of $\mathbb{R}^{m(n-1)}$ $\left\langle\xi_{k+1}, \ldots, \xi_{n-1}\right\rangle$ and the vectors of the two basis satisfy, by definition, the conditions

$$
\eta_{i} \xi_{j}=0 \quad i=1, \ldots, k \quad j=k+1, \ldots, n-1 .
$$

Hence, we can conclude that there exist vectors $\xi_{k+1}, \ldots, \xi_{n-1} \in \mathbb{R}^{n-1}$ such that $\bar{F} \in \operatorname{Ker} \varphi_{\#}$ if and only if $\bar{F} \xi_{i}=0$ for each $i=k+1, \ldots, n-1$.

Since $t \mapsto \varphi_{\#}(t, \bar{F})$ is decreasing on $(0,1)$ and it is coercive on $(0, \min f)$, there exist $0 \leq \min f \leq t_{1} \leq \ldots \leq t_{k} \leq t_{k+1} \leq \ldots \leq t_{n-1} \leq 1$ and $\xi_{k+1}, \ldots, \xi_{n-1} \in$ $\mathbb{R}^{n-1}$ such that

(i) $\varphi_{\#}(t, \bar{F})$ is coercive on $\left(0, t_{1}\right)$;

(ii) for each $k=1, \ldots, n-2 \varphi_{\#}(t, \bar{F})=0$ if and only if $\bar{F} \xi_{i}=0$

for $i=k+1, \ldots, n-1$ on $\left(t_{k}, t_{k+1}\right)$;

(iii) $\varphi_{\#}(t, \bar{F})=0$ on $\left(t_{n-1}, 1\right)$.

Definition 4.5 We define the 'degenerate weighted Sobolev Space' $\mathrm{W}_{\psi}^{1, p}\left(\Omega ; \mathbb{R}^{m}\right)$ as the space of functions $u \in \mathrm{L}^{p}\left(\Omega ; \mathbb{R}^{m}\right)$ such that

(i) $D_{n} u \in \mathrm{L}_{\text {loc }}^{p}\left(\Omega ; \mathbb{R}^{m}\right)$;

(ii) $D_{\left(\xi_{i}, 0\right)} u \in \mathrm{L}_{\text {loc }}^{p}\left(\omega \times\left(-t_{i}, t_{i}\right) ; \mathbb{R}^{m}\right)$ for $i=1, \ldots, n-1$;

(iii) if $\Phi: \Omega \rightarrow \mathbb{M}^{m \times(n-1)}$ is any measurable function such that $\Phi \xi_{i}=D_{\left(\xi_{i}, 0\right)} u \in \mathrm{L}_{\text {loc }}^{p}\left(\omega \times\left(-t_{i}, t_{i}\right) ; \mathbb{R}^{m}\right)$ for $i=1, \ldots, n-1$, then

$$
\int_{\Omega} \psi\left(x_{n}, \Phi \mid D_{n} u\right) d x<+\infty .
$$

Clearly, the last integral is independent of the choice of $\Phi$; hence, it will be denoted by

$$
\int_{\Omega} \psi\left(x_{n}, D u\right) d x
$$

with a slight abuse of notation.

Remark 4.6 Note that in dimension 3 (i.e., $n=3$ ) the representation of the space $\mathrm{W}_{\psi}^{1, p}\left(\Omega ; \mathbb{R}^{m}\right)$ is particularly simple as, up to a rotation, we can assume that 
$\xi=e_{2}$. In this case, $\mathrm{W}_{\psi}^{1, p}\left(\Omega ; \mathbb{R}^{m}\right)$ is the space of functions $u \in \mathrm{L}^{p}\left(\Omega ; \mathbb{R}^{m}\right)$ such that

(i) $D_{3} u \in \mathrm{L}_{\mathrm{loc}}^{p}\left(\Omega ; \mathbb{R}^{m}\right)$;

(ii) $D_{2} u \in \mathrm{L}_{\mathrm{loc}}^{p}\left(\omega \times\left(-t_{2}, t_{2}\right) ; \mathbb{R}^{m}\right)$;

(iii) $D_{1} u \in \mathrm{L}_{\text {loc }}^{p}\left(\omega \times\left(-t_{1}, t_{1}\right) ; \mathbb{R}^{m}\right)$;

(iv) if $\Phi: \Omega \rightarrow \mathbb{M}^{m \times 2}, \Phi=\left(\Phi_{1}, \Phi_{2}\right)$ is any measurable function such that $\Phi_{2}=D_{2} u$ in $\omega \times\left(-t_{2}, t_{2}\right)$ and $\Phi_{1}=D_{1} u$ in $\omega \times\left(-t_{1}, t_{1}\right)$, then

$$
\int_{\Omega} \psi\left(x_{3}, \Phi \mid D_{3} u\right) d x<+\infty \text {. }
$$

Example 4.7 If $n=3$ and

$$
f\left(x_{1}, x_{2}\right)=\frac{1}{2}+\frac{1}{2} \sin ^{2}\left(x_{1}\right) \sin ^{2}\left(x_{2}\right),
$$

then $\varphi_{\#}(t, \bar{F})=\|\bar{F}\|^{p}$ if $|t|<1 / 2$ and 0 otherwise, so that $t_{1}=t_{2}=1 / 2$, and $\xi$ is any vector. If instead

$$
f\left(x_{1}, x_{2}\right)=\frac{1}{2}+\frac{1}{2} \sin ^{2}\left(x_{1}\right),
$$

then $t_{1}=1 / 2, t_{2}=1$ and $\xi=(0,1)$.
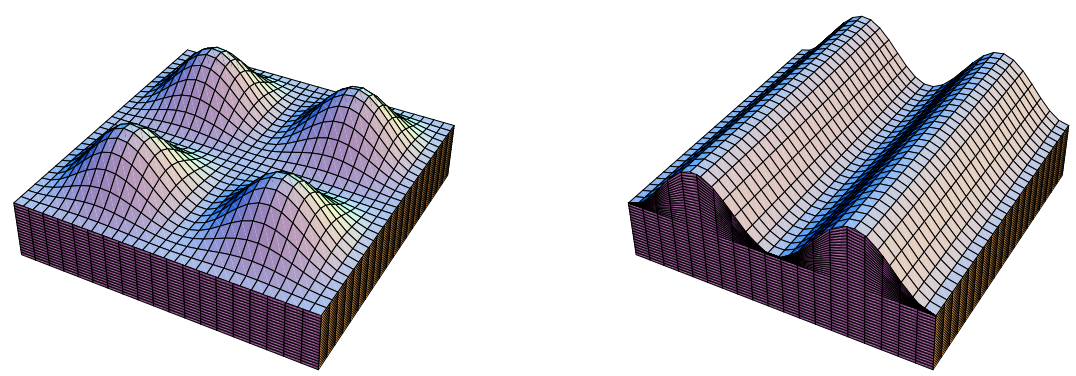

Figure 3: the oscillating profiles in Example 4.7

By using a convolution argument, we can improve Proposition 4.1 to give a characterization of the $\Gamma$-limit on the whole $\mathrm{W}_{\psi}^{1, p}\left(\Omega ; \mathbb{R}^{m}\right)$ and independent of the sequence $\left(\varepsilon_{j}\right)$. This result corresponds to Step 6 in Section 3, and its proof uses the convexity of $F \mapsto\|F\|^{p}$ in an essential way.

Proposition 4.8 Let $W=\|F\|^{p}$, and let $U$ be a open subset of $\Omega$. Then

(i) if $u \in \mathrm{L}^{p}\left(U ; \mathbb{R}^{m}\right) \backslash \mathrm{W}_{\psi}^{1, p}\left(U ; \mathbb{R}^{m}\right)$ then there exists the $\Gamma$-limit

$$
J_{0}(u, U)=\Gamma-\lim _{\varepsilon \rightarrow 0} J_{\varepsilon}(u, U)=+\infty ;
$$


(ii) if $u \in \mathrm{W}_{\psi}^{1, p}\left(\Omega ; \mathbb{R}^{m}\right)$ then there exists the $\Gamma$-limit

$$
J_{0}(u, U)=\Gamma-\lim _{\varepsilon \rightarrow 0} J_{\varepsilon}(u, U)=\int_{U} \psi\left(x_{n}, D u\right) d x .
$$

Proof. We only outline the proof, as it closely follows that of [6] Theorem 14.8 , and details can be found therein.

Fix $u \in \mathrm{L}^{p}\left(\Omega ; \mathbb{R}^{m}\right)$ and $U$ an open subset of $\Omega$. In order to compute $J_{0}(u, U)$ it is sufficient to show that from every sequence $\left(\varepsilon_{j}\right)$ we can extract a subsequence $\left(\varepsilon_{j_{k}}\right)$ such that the $\Gamma$-limit along $\left(\varepsilon_{j_{k}}\right)$ exists and is independent of the subsequence.

We fix a sequence $\left(\varepsilon_{j}\right)$. By Theorem 4.3 the thesis of Proposition 4.1 holds with $\psi$ in the place of $\varphi$. Upon possibly extracting a further subsequence, we may also assume that there exists the limit

$$
J_{0}(u, U)=\Gamma-\lim _{j \rightarrow+\infty} J_{\varepsilon_{j}}(u, U) .
$$

Let $\left(\rho_{j}\right)$ be a sequence of mollifiers with $\operatorname{spt} \rho_{j} \subset B\left(0, \frac{1}{j}\right) \subset \mathbb{R}^{n-1}$, and define

$$
u_{j}(x)=\int_{B\left(0, \frac{1}{j}\right)} \rho_{j}(y) u\left(x_{\alpha}-y, x_{n}\right) d y .
$$

By the convexity of $J_{0}$ and its translation-invariance properties, we have $J_{0}\left(u_{j}, U^{\prime}\right) \leq J_{0}(u, U)$ for all $U^{\prime} \subset \subset U$ such that $U^{\prime} \subset(y, 0)+U$ for all $y \in \operatorname{spt} \rho_{j}$. By the convexity of $\psi$ the functional $v \mapsto \int_{U^{\prime}} \psi\left(x_{n}, D v\right) d x$ (if $v \in \mathrm{L}^{p}\left(U^{\prime} ; \mathbb{R}^{m}\right) \backslash$ $\mathrm{W}_{\psi}^{1, p}\left(U^{\prime} ; \mathbb{R}^{m}\right)$ this integral is set equal to $\left.+\infty\right)$ is lower semicontinuous with respect to the $\mathrm{L}^{p}\left(U^{\prime} ; \mathbb{R}^{m}\right)$ convergence. Hence, we have

$$
\int_{U^{\prime}} \psi\left(x_{n}, D u\right) d x \leq \liminf _{j \rightarrow+\infty} \int_{U^{\prime}} \psi\left(x_{n}, D u_{j}\right) d x \leq J_{0}(u, U) .
$$

By the arbitrariness of $U^{\prime}$ we get

$$
\int_{U} \psi\left(x_{n}, D u\right) d x \leq J_{0}(u, U),
$$

and in particular that $J_{0}(u, U)=+\infty$ if $u \in \mathrm{L}^{p}\left(U ; \mathbb{R}^{m}\right) \backslash \mathrm{W}_{\psi}^{1, p}\left(U ; \mathbb{R}^{m}\right)$, so that (i) is proved.

Let now $u \in \mathrm{W}_{\psi}^{1, p}\left(\Omega ; \mathbb{R}^{m}\right)$. We first assume that $U \subset \subset U^{\prime} \subset \subset \Omega$. By using the lower semicontinuity of $J_{0}$ and Jensen's inequality, we have

$$
\begin{aligned}
J_{0}(u, U) & \leq \liminf _{j \rightarrow+\infty} J_{0}\left(u_{j}, U\right)=\liminf _{j \rightarrow+\infty} \int_{U} \psi\left(x_{n}, D u_{j}\right) d x \\
& \leq \liminf _{j \rightarrow+\infty} \int_{U} \int_{B\left(0, \frac{1}{j}\right)} \rho_{j}(y) \psi\left(x_{n}, D u(x-(y, 0))\right) d x d y \\
& =\liminf _{j \rightarrow+\infty} \int_{B\left(0, \frac{1}{j}\right)} \rho_{j}(y) \int_{U+(y, 0)} \psi\left(x_{n}, D u\right) d x d y \\
& \leq \liminf _{j \rightarrow+\infty} \int_{B\left(0, \frac{1}{j}\right)} \rho_{j}(y) d y \int_{U^{\prime}} \psi\left(x_{n}, D u\right) d x=\int_{U^{\prime}} \psi\left(x_{n}, D u\right) d x .
\end{aligned}
$$


By the arbitrariness of $U^{\prime}$ we then get

$$
J_{0}(u, U) \leq \int_{U} \psi\left(x_{n}, D u\right) d x,
$$

so that (ii) follows by taking (4.9) into account.

Finally, for arbitrary $U$, note that if $u \in \mathrm{W}_{\psi}^{1, p}\left(\Omega ; \mathbb{R}^{m}\right)$ then it can be approximated by a sequence $\left(v_{j}\right)$ of functions in $\mathrm{W}^{1, p}\left(\Omega ; \mathbb{R}^{m}\right)$ such that $\int_{\Omega} \psi\left(x_{n}, D v_{j}\right) d x$ are equi-bounded (we may use e.g. the argument in the proof of [15] Section 4.2 Theorem 3); hence, by the lower semicontinuity of $J^{\prime \prime}=\Gamma-\lim \sup _{j} J_{\varepsilon_{j}}$, we have $J^{\prime \prime}(u)<+\infty$. This fact implies (as in e.g. [6] Section 11.2) that $J^{\prime \prime}$ is inner-regular; i.e.,

$$
J^{\prime \prime}(u, U)=\sup \left\{J^{\prime \prime}(u, V): V \subset \subset U\right\} .
$$

Since (ii) holds with $V$ in the place of $U$ we easily get the thesis.

The following proposition clarifies the structure of $\mathrm{W}_{\psi}^{1, p}$, and implies that the restrictions of functions $u \in \mathrm{W}_{\psi}^{1, p}\left(\Omega ; \mathbb{R}^{m}\right)$ to relatively compact subsets of $\omega \times\left(t_{k}, t_{k+1}\right)$ are characterized as those functions having directional derivatives $D_{k+1}, \ldots, D_{n} p$-summable.

Proposition 4.9 Let $k=1, \ldots, n-2$ and $s \in\left(t_{k}, t_{k+1}\right)$. There exist two positive constants $\alpha_{k}(s)$ and $\beta_{k}$ such that

$$
\alpha_{k}(s)\left(\sum_{i=k+1}^{n-1}\left|\bar{F} \xi_{i}\right|^{p}+\left|F_{n}\right|^{p}\right) \leq \psi(t, F) \leq \beta_{k}\left(\sum_{i=k+1}^{n-1}\left|\bar{F} \xi_{i}\right|^{p}+\left|F_{n}\right|^{p}\right)
$$

for all $F \in \mathbb{M}^{m \times n}$ and $t \in\left(t_{k}, s\right]$.

Proof. Since $\bar{F} \mapsto \varphi_{\#}(t, \bar{F})$ is positively homogeneous of degree $p$ and convex, if $t \in\left(t_{k}, t_{k+1}\right)$ we easily deduce that

$$
\varphi_{\#}(t, \bar{F}) \leq c \sum_{i=k+1}^{n-1} \varphi_{\#}\left(t, \Xi_{i}\right)\left|\bar{F} \xi_{i}\right|^{p}
$$

where

$$
\Xi_{i}=\left(\begin{array}{c}
\xi_{i} \\
0 \\
\vdots \\
0
\end{array}\right)
$$

If we denote

$$
\beta_{k}^{\prime}=\max _{i=k+1, \ldots, n-1} \sup _{t \in[0,1)} c \varphi_{\#}\left(t, \Xi_{i}\right)
$$


then

$$
\varphi_{\#}(t, \bar{F}) \leq \beta_{k}^{\prime} \sum_{i=k+1}^{n-1}\left|\bar{F} \xi_{i}\right|^{p} .
$$

On the other hand we have that

$$
\begin{aligned}
\frac{\varphi_{\#}(t, \bar{F})}{\sum_{i=k+1}^{n-1}\left|\bar{F} \xi_{i}\right|^{p}} & \geq c \frac{\varphi_{\#}\left(t,\left(\bar{F} \xi_{k+1}, \ldots, \bar{F} \xi_{n-1}\right)\right)}{\left\|\left(\bar{F} \xi_{k+1}, \ldots, \bar{F} \xi_{n-1}\right)\right\|^{p}} \\
& \geq c \inf \left\{\varphi_{\#}(t, G): G \in S^{n-1} \cap \operatorname{Ker} \varphi_{\#}^{\perp}\right\}
\end{aligned}
$$

by p-homogeneity. Note that $t \mapsto c \inf \left\{\varphi_{\#}(t, G): G \in S^{n-1} \cap \operatorname{Ker} \varphi_{\#}^{\perp}\right\}=c(t)$ is decreasing on $(0,1)$ and

$$
\inf _{t \in\left(t_{k}, s\right]} c(t)=\alpha_{k}^{\prime}(s)>0,
$$

so that we get

$$
\varphi_{\#}(t, \bar{F}) \geq \alpha_{k}^{\prime}(s) \sum_{i=k+1}^{n-1}\left|\bar{F} \xi_{i}\right|^{p} .
$$

Let

$$
\alpha_{k}(s)=\min \left\{\alpha_{k}^{\prime}(s), \inf _{t \in\left(t_{k}, s\right]} \mathcal{L}_{n-1}\left(E_{t} \cap(0,1)^{n-1}\right)\right\}
$$

and

$$
\beta_{k}=\max \left\{\beta_{k}^{\prime}, 1\right\} \text {, }
$$

then (4.11) follows by Theorem 4.3, 4.12) and (4.13).

Proposition 4.10 Fix $t \in\left(t_{k}, t_{k+1}\right)$, for $k=0, \ldots, n-1\left(t_{0}=0, t_{n}=1\right)$. If $\psi$ is given by (4.7) then

$$
\begin{aligned}
\psi(t, F)=\min \left\{\int_{(0,1)^{n} \cap\left(E_{t} \times(0,1)\right)}\|D w\|^{p} d x:\right. & \\
& \left.w \in \mathrm{W}_{\text {loc }}^{1, p}\left(E_{t} \times(0,1) ; \mathbb{R}^{m}\right), w-F x 1 \text {-periodic }\right\} .
\end{aligned}
$$

Proof. Let $w$ be a test function for the minimum problem above, then

$$
\begin{aligned}
& \int_{(0,1)^{n} \cap\left(E_{t} \times(0,1)\right)}\|D w\|^{p} d x \\
= & \int_{(0,1)^{n} \cap\left(E_{t} \times(0,1)\right)}\left\|D_{\alpha} w\right\|^{p} d x+\int_{(0,1)^{n} \cap\left(E_{t} \times(0,1)\right)}\left|D_{n} w\right|^{p} d x \\
\geq & \int_{0}^{1} \min \left\{\int_{E_{t} \cap(0,1)^{n-1}}\|D v\|^{p} d x_{\alpha}:\right.
\end{aligned}
$$




$$
\begin{array}{r}
\left.v \in \mathrm{W}_{\text {loc }}^{1, p}\left(E_{t} ; \mathbb{R}^{m}\right), v-\bar{F} x_{\alpha} \text { 1-periodic }\right\} d x_{n} \\
+\int_{E_{t} \cap(0,1)^{n-1}}\left(\int_{0}^{1}\left|D_{n} w\right|^{p} d x_{n}\right) d x_{\alpha} \\
\geq \varphi_{\#}(t, \bar{F})+\mathcal{L}_{n-1}\left(E_{t} \cap(0,1)^{n-1}\right)\left|F_{n}\right|^{p}=\psi(t, F)
\end{array}
$$

by Jensen's inequality and the description of $\varphi_{\#}$ (see Theorem 4.2); hence,

$$
\begin{aligned}
\psi(t, F) \leq \min \left\{\int_{(0,1)^{n} \cap E_{t} \times(0,1)}\|D w\|^{p} d x:\right. & \\
& \left.w \in \mathrm{W}_{\text {loc }}^{1, p}\left(E_{t} \times(0,1) ; \mathbb{R}^{m}\right), w-F x \text { 1-periodic }\right\}
\end{aligned}
$$

by Theorem 4.3 .

Conversely, given a function $v$ such that $v-\bar{F} x_{\alpha}$ is 1-periodic, we can construct a test function $w$, such that $w-F x$-periodic, as

$$
w(x)=v\left(x_{\alpha}\right)-F_{n} x_{n} .
$$

We then have

$$
\begin{aligned}
& \int_{(0,1)^{n} \cap\left(E_{t} \times(0,1)\right)}\|D w\|^{p} d x \\
&= \int_{(0,1)^{n} \cap\left(E_{t} \times(0,1)\right)}\left(\left\|D_{\alpha} v\right\|^{p}+\left|F_{n}\right|^{p}\right) d x \\
&= \int_{E_{t} \cap(0,1)^{n-1}}\left\|D_{\alpha} v\right\|^{p} d x_{\alpha}+\mathcal{L}_{n-1}\left(E_{t} \cap(0,1)^{n-1}\right)\left|F_{n}\right|^{p} \\
& \geq \min \left\{\int_{(0,1)^{n} \cap\left(E_{t} \times(0,1)\right)}\|D w\|^{p} d x:\right. \\
&\left.w \in \mathrm{W}_{\text {loc }}^{1, p}\left(E_{t} \times(0,1) ; \mathbb{R}^{m}\right), w-F x \text { 1-periodic }\right\}
\end{aligned}
$$

and hence the converse inequality

$$
\begin{aligned}
& \psi(t, F)= \min \left\{\int_{E_{t} \cap(0,1)^{n-1}}\|D v\|^{p} d x_{\alpha}:\right. \\
&\left.v \in \mathrm{W}_{\text {loc }}^{1, p}\left(E_{t} ; \mathbb{R}^{m}\right), v-\bar{F} x_{\alpha} \text { 1-periodic }\right\} \\
&+\mathcal{L}_{n-1}\left(E_{t} \cap(0,1)^{n-1}\right)\left|F_{n}\right|^{p} \\
& \geq \min \left\{\int_{(0,1)^{n} \cap\left(E_{t} \times(0,1)\right)}\|D w\|^{p} d x:\right. \\
&\left.\quad w \in \mathrm{W}_{\text {loc }}^{1, p}\left(E_{t} \times(0,1) ; \mathbb{R}^{m}\right), w-F x \text { 1-periodic }\right\}
\end{aligned}
$$

is obtained as desired. 
Now we can turn our attention to the case with a general $W$. Now that a natural domain for the limit functional is defined, we can easily state and prove a compactness result that partly improves Proposition 4.1.

Theorem 4.11 Let $J_{\varepsilon}$ be given by (4.4). Then for every sequence $\left(\varepsilon_{j}\right)$ of positive numbers converging to 0 there exists a subsequence (not relabeled) such that the $\Gamma$-limit

$$
J_{0}(u, U)=\Gamma-\lim _{j \rightarrow+\infty} J_{\varepsilon_{j}}(u, U)
$$

exists for all $u \in \mathrm{W}_{\psi}^{1, p}\left(\Omega ; \mathbb{R}^{m}\right)$ and $U$ open subsets of $\Omega$. Moreover $J_{0}(u, \cdot)$ is the restriction of a Borel measure to $\mathcal{A}(\Omega)$.

Proof. By (4.1) and Proposition 4.8 we deduce the condition

$$
\Gamma-\limsup _{\varepsilon \rightarrow 0} J_{\varepsilon}(u, U) \leq \beta \int_{U}\left(1+\psi\left(x_{n}, D u\right)\right) d x
$$

if $u \in \mathrm{W}_{\psi}^{1, p}\left(U ; \mathbb{R}^{m}\right)$ and $U$ is an open subset of $\Omega$. Then, we can follow the Steps 1-3 in Section 3 to prove the compactness of $\left(J_{\varepsilon}\right)$ and that $J_{0}(u, \cdot)$ is the restriction of a Borel measure to $\mathcal{A}(\Omega)$.

\subsection{Homogenization of cylindrical domains}

It remains now to extend the integral representation of Proposition 4.1 and characterize its integrand. We first deal with the case of 'cylindrical' domains; i.e., we consider $\chi_{E}$ in place of $f$, with $E$ a 1 -periodic open subset of $\mathbb{R}^{n-1}$.

Let $t_{1}, \ldots, t_{n-1}$ be the points in $(0,1)$ introduced to characterize the 'degenerate weighted Sobolev Space' in Definition 4.5. Since in the following we will choose $E=E_{t}\left(E_{t}\right.$ defined as $\left.\left\{x_{\alpha}: f\left(x_{\alpha}\right)>|t|\right\}\right)$ we introduce the following notation: with fixed $t \in(0,1), t \neq t_{k}$ for $k=1, \ldots, n-1$, consider the set $E_{t}$ and the functional

$$
J_{\varepsilon}^{t}(u, U)= \begin{cases}\int_{\Omega_{\varepsilon} \cap U_{\varepsilon}} W\left(\frac{x_{\alpha}}{\varepsilon}, D u\right) d x & \text { if } u \in \mathrm{W}^{1, p}\left(\Omega_{\varepsilon} \cap U_{\varepsilon} ; \mathbb{R}^{m}\right) \\ +\infty & \text { otherwise, }\end{cases}
$$

where $U_{\varepsilon}=U \cap\left(\varepsilon E_{t} \times(-1,1)\right)$. Note that the integrand of $J_{\varepsilon}^{t}$ satisfies the following growth conditions

$$
\gamma g(x, A) \leq \chi_{E_{t} \times(-1,1)} W\left(x_{\alpha}, A\right) \leq \beta(1+g(x, A))
$$

where $g(x, A)=\chi_{E_{t} \times(-1,1)}(x)\|A\|^{p}$ is obviously 1-periodic in $x$, convex in $A$ and satisfying

$$
0 \leq g(x, A) \leq 1+\|A\|^{p} \text { and } g(x, 2 A) \leq c(1+g(x, A))
$$

for all $A \in \mathbb{M}^{m \times n}$. 
Remark 4.12 Note that if we fix $t \in\left(t_{k-1}, t_{k}\right)$ and consider $\chi_{E_{t}}$ in place of $f$ then $\mathrm{W}_{\psi}^{1, p}\left(\Omega ; \mathbb{R}^{m}\right)$ turns out to be the space

$$
\begin{gathered}
\mathrm{W}_{k}^{1, p}\left(\Omega ; \mathbb{R}^{m}\right)=\left\{u \in \mathrm{L}^{p}\left(\Omega ; \mathbb{R}^{m}\right): D_{n} u \in \mathrm{L}^{p}\left(\Omega ; \mathbb{R}^{m}\right), D_{\xi_{i}} u \in \mathrm{L}^{p}\left(\Omega ; \mathbb{R}^{m}\right)\right. \\
i=k, \ldots, n-1\}
\end{gathered}
$$

if $k=1, \ldots, n-1$, and

$$
\mathrm{W}_{n}^{1, p}\left(\Omega ; \mathbb{R}^{m}\right)=\left\{u \in \mathrm{L}^{p}\left(\Omega ; \mathbb{R}^{m}\right): D_{n} u \in \mathrm{L}^{p}\left(\Omega ; \mathbb{R}^{m}\right)\right\} .
$$

if $k=n$.
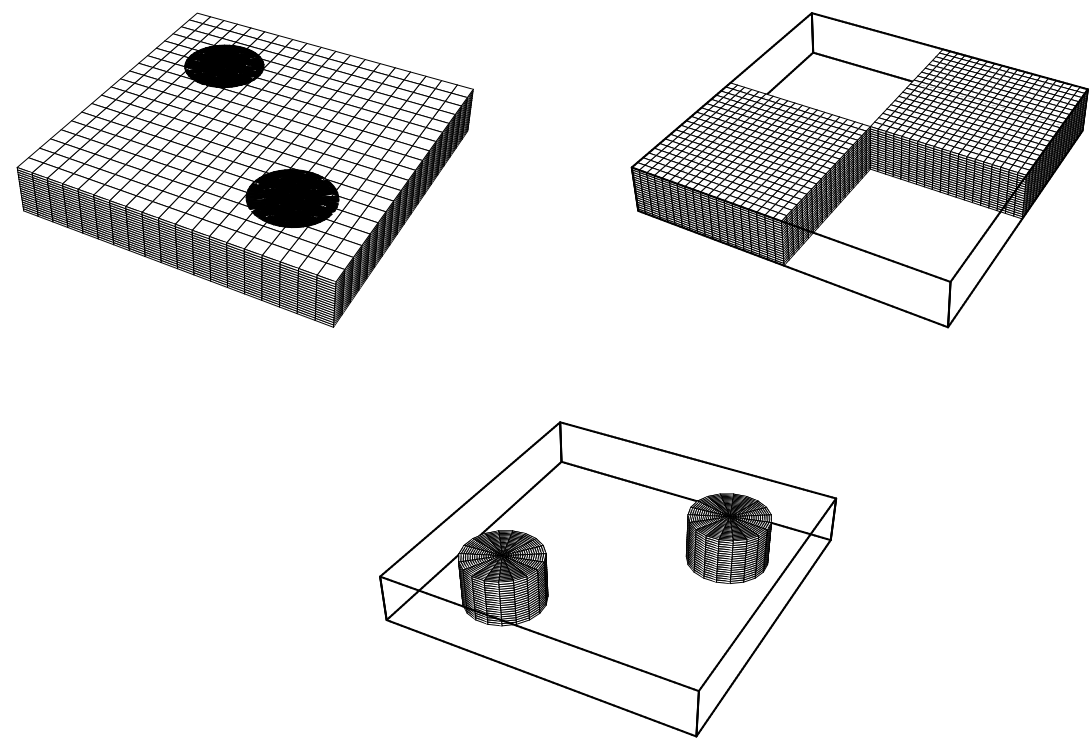

Figure 4: cylindrical domains $E_{t} \times(-1,1)$ related to the function $f$ in Figure 1 for different values of $t$

Theorem 4.13 Let $t \in\left(t_{k-1}, t_{k}\right)$ and let $J_{\varepsilon}^{t}(\cdot, U)$ be defined by 4.15). Then the Г-limit

$$
J_{0}^{t}(u, U)=\int_{U} W_{\mathrm{hom}}^{t}(D u) d x
$$

exists for each $u \in \mathrm{W}_{k}^{1, p}\left(\Omega ; \mathbb{R}^{m}\right)$ and $U$ open subset of $\Omega$, where $W_{\mathrm{hom}}^{t}$ is given by

$$
\begin{gathered}
W_{\text {hom }}^{t}(A)=\lim _{T \rightarrow+\infty} \inf \left\{\frac{1}{T^{n}} \int_{(0, T)^{n}} \chi_{E_{t}}\left(x_{\alpha}\right) W\left(x_{\alpha}, A+D u(x)\right) d x:\right. \\
\left.u \in \mathrm{W}_{0}^{1, p}\left((0, T)^{n} ; \mathbb{R}^{m}\right)\right\}
\end{gathered}
$$


for all $A \in \mathbb{M}^{m \times n}$.

Proof. By taking Theorem 4.11 into account with $\chi_{E_{t}}$ in the place of $f$, and repeating word for word the proof of the integral representation theorem [6] Theorem 9.1 , replacing $\mathrm{W}^{1, p}\left(\Omega ; \mathbb{R}^{m}\right)$ by $\mathrm{W}_{k}^{1, p}\left(\Omega ; \mathbb{R}^{m}\right)$, we obtain an integral representation on the whole $\mathrm{W}_{k}^{1, p}\left(\Omega ; \mathbb{R}^{m}\right)$. The integrand of this representation must coincide with the function $\varphi=\varphi\left(x_{n}, F\right)$ provided by Proposition 4.1 with $\chi_{E_{t}}$ in the place of $f$. Since the functionals are clearly invariant by translations in the direction $x_{n}$ we have indeed $\varphi=\varphi(F)$. To prove the asymptotic formula we can repeat the proof of Proposition 21.12 in [6].

\subsection{The general case}

We can eventually proceed to dealing with the general case.

Proposition 4.14 Let $J_{\varepsilon}$ be given by (4.4). Then the $\Gamma$-limit

$$
J_{0}(u, U)=\Gamma-\lim _{\varepsilon \rightarrow 0} J_{\varepsilon}(u, U)
$$

exists for all $u \in \mathrm{W}_{\psi}^{1, p}\left(\Omega ; \mathbb{R}^{m}\right)$ and $U$ open subsets of $\Omega$. Moreover, for such $u$ we have

$$
J_{0}(u, U)=\int_{U} \varphi\left(x_{n}, D u\right) d x,
$$

where $\varphi$ is given by Proposition 4.1.

Proof. We have to extend the representation of $J_{0}$ given by Proposition 4.1 to $\mathrm{W}_{\psi}^{1, p}\left(\Omega ; \mathbb{R}^{m}\right)$. Note that $\varphi$ is a Carathéodory function (see [6] Theorem 9.1, Step 3). As explained in Step 3 of Section 3, a crucial argument used to obtain an integral representation result is the continuity in $\mathrm{W}_{\psi}^{1, p}\left(\Omega ; \mathbb{R}^{m}\right)$ of the functional

$$
u \mapsto \int_{U} \varphi\left(x_{n}, D u\right) d x
$$

along some strongly converging sequences of piecewise-affine functions. We only prove this property, as the rest of the proof follows exactly that of [6] Theorem 9.1 (Steps 1-3, 5 and 6; the proof below replaces Step 4).

Let $U=\bigcup_{k=0}^{n-1} U_{k}$ where $U_{k} \subset \subset \omega \times\left(t_{k}, t_{k+1}\right),\left(t_{0}=0, t_{n}=1\right)$; we can find functions $u_{j} \in \mathrm{W}_{\psi}^{1, p}\left(\Omega ; \mathbb{R}^{m}\right)$ such that their restrictions to $U$ are piecewise affine and $u_{j}, D_{n} u_{j}$ converge strongly to $u, D_{n} u$ in $L^{p}\left(U ; \mathbb{R}^{m}\right)$, respectively, while $D_{\left(\xi_{i}, 0\right)} u_{j}$ converge strongly to $D_{\left(\xi_{i}, 0\right)} u$ in $\mathrm{L}^{p}\left(U_{i} ; \mathbb{R}^{m}\right)$.

We will use some estimates deriving from the inequality $\varphi(t, F) \leq \beta(1+$ $\psi(t, F)$ ), which follows trivially from (4.1). By Proposition 4.9 we have that

$$
\psi\left(x_{n}, D u\right) \leq \beta_{k}\left(\sum_{i=k+1}^{n-1}\left|D_{\left(\xi_{i}, 0\right)} u\right|^{p}+\left|D_{n} u\right|^{p}\right)
$$




$$
\psi\left(x_{n}, D u_{j}\right) \leq \beta_{k}\left(\sum_{i=k+1}^{n-1}\left|D_{\left(\xi_{i}, 0\right)} u_{j}\right|^{p}+\left|D_{n} u_{j}\right|^{p}\right)
$$

on $\omega \times\left(t_{k}, t_{k+1}\right)$. Note that by 4.14

$$
\begin{aligned}
\int_{U} \varphi\left(x_{n}, D u_{j}\right) d x \leq & \sum_{k=0}^{n-2} \int_{U_{k} \cap \omega \times\left(t_{k}, t_{k+1}\right)} \beta\left(1+\sum_{i=k+1}^{N} \beta_{k}\left|D_{\left(\xi_{i}, 0\right)} u_{j}\right|^{p}\right) d x \\
& +\beta \int_{U} \beta_{k}\left|D_{n} u_{j}\right|^{p} d x .
\end{aligned}
$$

If we use the continuity of $\varphi$ in the second variable and apply Fatou's lemma to the sequences

$$
\begin{aligned}
\beta \int_{U} \beta_{k}\left|D_{n} u_{j}\right|^{p} d x & +\sum_{k=0}^{n-2} \int_{U_{k} \cap \omega \times\left(t_{k}, t_{k+1}\right)} \beta\left(1+\sum_{i=k+1}^{n-1} \beta_{k}\left|D_{\left(\xi_{i}, 0\right)} u_{j}\right|^{p}\right) d x \\
& \pm \int_{U} \varphi\left(x_{n}, D u_{j}\right) d x
\end{aligned}
$$

we get that

$$
\int_{U} \varphi\left(x_{n}, D u\right) d x=\lim _{j \rightarrow+\infty} \int_{U} \varphi\left(x, D u_{j}\right) d x .
$$

Hence, we have proved the integral representation for sets of the type $U=\bigcup_{k=0}^{n-1} U_{k}$ where $U_{k} \subset \subset \omega \times\left(t_{k}, t_{k+1}\right)$. A symmetric argument applies to the case where $U=\bigcup_{k=0}^{n-1} U_{k}$, with $U_{k} \subset \subset \omega \times\left(-t_{k+1},-t_{k}\right)$. Since $J_{0}(u, \cdot)$ is a measure absolutely continuous with respect to Lebesgue measure, we conclude that the integral representation holds for all open subsets $U$ of $\Omega$.

Finally, the oscillating-boundary homogenization theorem reads as follows.

Theorem 4.15 Let $J_{\varepsilon}$ be given by (4.2). Then the $\Gamma$-limit

$$
J_{0}(u)=\Gamma-\lim _{\varepsilon \rightarrow 0} J_{\varepsilon}(u)
$$

exists for all $u \in \mathrm{L}^{p}\left(\Omega ; \mathbb{R}^{m}\right)$, and we have

$$
J_{0}(u)= \begin{cases}\int_{\Omega} W_{\mathrm{hom}}\left(\left|x_{n}\right|, D u\right) d x & \text { if } u \in \mathrm{W}_{\psi}^{1, p}\left(\Omega ; \mathbb{R}^{m}\right) \\ +\infty & \text { otherwise, }\end{cases}
$$

where $W_{\mathrm{hom}}(t, A)=W_{\mathrm{hom}}^{t}(A)$ for a.e. $t \in(0,1)$, and $W_{\mathrm{hom}}^{t}$ is given by Theorem 4.13. Moreover, if $u \in \mathrm{W}^{1, p}\left(\Omega ; \mathbb{R}^{m}\right)$ there exists a family $\left(u_{\varepsilon}\right)$ converging to $u$ in $\mathrm{L}^{p}\left(\Omega ; \mathbb{R}^{m}\right)$, such that $u-u_{\varepsilon}$ has compact support in $\Omega$ and $J_{0}(u)=\lim _{\varepsilon \rightarrow 0} J_{\varepsilon}\left(u_{\varepsilon}\right)$. 
Proof. It is sufficient to compute the $\Gamma$-limit for $u \in \mathrm{W}_{\psi}^{1, p}\left(\Omega ; \mathbb{R}^{m}\right)$, since by comparison with Proposition $4.8(\mathrm{i})$ we immediately have $J_{0}(u)=+\infty$ if $u \notin$ $\mathrm{W}_{\psi}^{1, p}\left(\Omega ; \mathbb{R}^{m}\right)$. Let $\varphi$ be given by Proposition 4.1 ; it remains to prove that $\varphi$ satisfies an asymptotic formula.

Let $x_{n}>0$, let $0<\rho<x_{n}$ and consider the functionals 4.15) with $t=x_{n}-\rho$ and $t=x_{n}$ so that

$$
\begin{aligned}
J_{\varepsilon}^{x_{n}-\rho}\left(A x,(0,1)^{n-1} \times\left(x_{n}-\rho, x_{n}\right)\right) \\
\quad \geq \int_{(0,1)^{n-1} \times\left(x_{n}-\rho, x_{n}\right)} \chi_{E_{y_{n}}}\left(\frac{y_{\alpha}}{\varepsilon}\right) W\left(\frac{x_{\alpha}}{\varepsilon}, A\right) d y \\
\quad \geq J_{\varepsilon}^{x_{n}}\left(A x,(0,1)^{n-1} \times\left(x_{n}-\rho, x_{n}\right)\right) .
\end{aligned}
$$

By Theorem 4.13

$$
\begin{aligned}
\rho W_{\mathrm{hom}}^{x_{n}-\rho}(A) & \geq \Gamma-\lim _{\varepsilon \rightarrow 0} J_{\varepsilon}\left(A x,(0,1)^{n-1} \times\left(x_{n}-\rho, x_{n}\right)\right) \\
& \geq \rho W_{\mathrm{hom}}^{x_{n}}(A) .
\end{aligned}
$$

Taking into account that

$$
\Gamma-\lim _{\varepsilon \rightarrow 0} J_{\varepsilon}\left(A x,(0,1)^{n-1} \times\left(x_{n}-\rho, x_{n}\right)\right)=\int_{(0,1)^{n-1} \times\left(x_{n}-\rho, x_{n}\right)} \varphi\left(y_{n}, A\right) d y
$$

we get

$$
W_{\mathrm{hom}}^{x_{n}-\rho}(A) \leq \frac{1}{\rho} \int_{\left(x_{n}-\rho, x_{n}\right)} \varphi\left(y_{n}, A\right) d y_{n} \leq W_{\mathrm{hom}}^{x_{n}}(A) .
$$

Since $t \mapsto W_{\text {hom }}^{t}(A)$ and $t \mapsto \varphi(t, A)$ are decreasing functions on $(0,1)$, there exists a subset $M$ of $(0,1),|M|=0$, such that they are continuous on $(0,1) \backslash M$; hence, by passing to the limit as $\rho \rightarrow 0$ we get

$$
\varphi\left(x_{n}, A\right)=W_{\mathrm{hom}}^{x_{n}}(A)
$$

for every $x_{n} \in(0,1) \backslash M$. For $x_{n}<0$ it suffices to apply a symmetric argument.

The last statement follows by a well-known argument of stability of $\Gamma$-convergence by compatible boundary data due to De Giorgi (see [6] Section 11.3).

\section{Thin films with fast-oscillating profile}

In this section we establish the second goal of the paper; that is, to prove that the $\Gamma$-limit of functionals $E_{\varepsilon, \delta}$ as in (1.1) when $\varepsilon \rightarrow 0$ and $\delta<<\varepsilon$, is given by first applying the theory constructed in the previous section with $\varepsilon$ as a parameter and letting $\delta \rightarrow 0$, and subsequently letting $\varepsilon \rightarrow 0$. The final result can be summarized as follows, in a $n$-dimensional setting. 
Theorem 5.1 Let $f: \mathbb{R}^{n-1} \rightarrow[0,1]$ be a 1 -periodic lower semicontinuous function with $0<\min f \leq \sup f=1$, let $W: \mathbb{M}^{m \times n} \rightarrow[0,+\infty)$ be a convex function satisfying

$$
\gamma|F|^{p} \leq W(F) \leq \beta\left(1+|F|^{p}\right)
$$

for all $F \in \mathbb{M}^{m \times n}$ and for some $1<p<+\infty, 0<\gamma \leq \beta$. Let $\delta:(0,+\infty) \rightarrow$ $(0,+\infty)$ be such that

$$
\lim _{\varepsilon \rightarrow 0} \frac{\delta(\varepsilon)}{\varepsilon}=0 .
$$

Let $\omega$ be a bounded open subset of $\mathbb{R}^{n-1}$ and let $\Omega_{\varepsilon} \subset \omega \times(-1,1)$ be defined by

$$
\Omega_{\varepsilon}=\left\{x \in \mathbb{R}^{n}:\left|x_{n}\right|<f\left(\frac{x_{\alpha}}{\delta(\varepsilon)}\right), x_{\alpha} \in \omega\right\} .
$$

Define $E_{\varepsilon}: \mathrm{L}^{p}(\omega \times(-1,1)) \rightarrow[0,+\infty]$ by

$$
E_{\varepsilon}(u)= \begin{cases}\int_{\Omega_{\varepsilon}} W\left(D_{\alpha} u, \frac{1}{\varepsilon} D_{n} u\right) d x & \text { if } u_{\Omega_{\varepsilon}} \in \mathrm{W}^{1, p}\left(\Omega_{\varepsilon} ; \mathbb{R}^{m}\right) \\ +\infty & \text { otherwise. }\end{cases}
$$

Then the $\Gamma$-limit as $\varepsilon \rightarrow 0$ of $E_{\varepsilon}$ is given by

$E(u)= \begin{cases}\int_{\omega \times(-1,1)} \bar{W}_{\mathrm{hom}}\left(D_{\alpha} u\right) d x & \text { if } u \in \mathrm{W}^{1, p}\left(\omega \times(-1,1) ; \mathbb{R}^{m}\right) \text { and } D_{n} u=0 \\ +\infty & \text { otherwise, }\end{cases}$

where $\bar{W}_{\mathrm{hom}}: \mathbb{M}^{m \times(n-1)} \rightarrow[0,+\infty)$ is given by

$$
\bar{W}_{\text {hom }}(\bar{F})=\int_{0}^{1} \inf _{F_{n}} W_{\text {hom }}\left(t, \bar{F} \mid F_{n}\right) d t,
$$

and $W_{\text {hom }}$ by

$$
\begin{array}{r}
W_{\text {hom }}(t, F)=\inf \left\{\int_{(0,1)^{n}} \chi_{E_{t}}\left(x_{\alpha}\right) W(F+D u(x)) d x:\right. \\
\left.u \in \mathrm{W}_{\text {loc }}^{1, p}\left(\mathbb{R}^{n} ; \mathbb{R}^{m}\right) 1 \text {-periodic }\right\}
\end{array}
$$

for all $t \in(0,1)$ and $F \in \mathbb{M}^{m \times n}$, where $E_{t}=\{f>t\}$.

\subsection{Proof of the result}

In order to simplify the proof without loosing sight of the main intricacies of the argument, we deal only with the case where $\varepsilon=1 / j$ and $\delta=\varepsilon^{2}$. The general case can be dealt with similarly, by introducing some error terms. We define, with a slight abuse of notation,

$$
\Omega_{k}=\left\{x \in \Omega:\left|x_{n}\right|<f\left(k x_{\alpha}\right)\right\}
$$


and for $k=j^{2}, j \in \mathbb{N}$

$$
E_{j}(u, U)=\int_{\Omega_{j^{2}} \cap U} W\left(D_{\alpha} u \mid j D_{n} u\right) d x
$$

for all $u_{\mid \Omega_{j^{2}} \cap U} \in \mathrm{W}^{1, p}\left(\Omega_{j^{2}} \cap U ; \mathbb{R}^{m}\right)$.

By the compactness result Theorem 2.5 in [8] we can suppose that there exists $W_{0}: \mathbb{M}^{m \times(n-1)} \rightarrow[0,+\infty)$ such that $E_{j}(u, U) \Gamma$-converge for all sets of the form $U=U^{\prime} \times(-1,1)$ or $U=U^{\prime} \times(0,1)$ to the functional given by

$$
E_{0}(u, U)= \begin{cases}\int_{U} W_{0}\left(D_{\alpha} u\right) d x & \text { if } u \in \mathrm{W}^{1, p}\left(U ; \mathbb{R}^{m}\right) \text { and } D_{n} u=0 \\ +\infty & \text { otherwise. }\end{cases}
$$

Proposition 5.2 For all $\bar{F} \in \mathbb{M}^{m \times(n-1)}$ define

$$
\begin{aligned}
\bar{W}_{\text {hom }}(\bar{F})=\inf \left\{\int_{(0,1)^{n}}\right. & W_{\text {hom }}\left(x_{n}, D u+\bar{F}\right) d x: \\
u & \left.\in \mathrm{W}_{\text {loc }}^{1, p}\left(\mathbb{R}^{n} ; \mathbb{R}^{m}\right), u 1 \text {-periodic in } x_{\alpha}\right\} .
\end{aligned}
$$

Then

$$
\bar{W}_{\text {hom }}(\bar{F})=\int_{0}^{1} \widetilde{W}_{\text {hom }}(t, \bar{F}) d t
$$

where

$$
\widetilde{W}_{\text {hom }}(t, \bar{F})=\inf _{F_{n}} W_{\text {hom }}\left(t, \bar{F} \mid F_{n}\right)
$$

and $\bar{F} \mapsto \widetilde{W}_{\text {hom }}(t, \bar{F})$ is convex.

Proof. It can be easily proved that $\bar{F} \mapsto \widetilde{W}_{\text {hom }}(t, \bar{F})$ is convex.

With fixed $\eta>0$, by the Measurable Selection Criterion (see e.g [12]), we can find $G_{n}(t)$ a measurable function such that

$$
W_{\text {hom }}\left(t, \bar{F} \mid G_{n}\right) \leq \inf _{F_{n}} W_{\text {hom }}\left(t, \bar{F} \mid F_{n}\right)+\eta .
$$

We can consider

$$
u\left(x_{\alpha}, x_{n}\right)=\int_{0}^{x_{n}} G_{n}(s) d s
$$

as test function in (5.7). We then get

$$
\bar{W}_{\text {hom }}(\bar{F}) \leq \int_{0}^{1} W_{\text {hom }}\left(x_{n}, \bar{F} \mid G_{n}\left(x_{n}\right)\right) d x_{n}
$$


and so

$$
\bar{W}_{\text {hom }}(\bar{F}) \leq \int_{0}^{1} \inf _{F_{n}} W_{\text {hom }}\left(t, \bar{F} \mid F_{n}\right) d t+\eta=\int_{0}^{1} \widetilde{W}_{\text {hom }}(t, \bar{F}) d t+\eta .
$$

Conversely,

$$
\begin{aligned}
& \bar{W}_{\text {hom }}(\bar{F}) \geq \inf \left\{\int_{(0,1)^{n}} \widetilde{W}_{\text {hom }}\left(x_{n}, D_{\alpha} u+\bar{F}\right) d x:\right. \\
&\left.u \in \mathrm{W}_{\text {loc }}^{1, p}\left((0,1)^{n} ; \mathbb{R}^{m}\right), u \text { 1-periodic in } x_{\alpha}\right\} \\
& \geq \int_{0}^{1}\left(\operatorname { i n f } \left\{\int_{(0,1)^{n-1}} \widetilde{W}_{\text {hom }}\left(t, D_{\alpha} u+\bar{F}\right) d x_{\alpha}:\right.\right. \\
&\left.\left.u_{\mid(0,1)^{n-1}} \in \mathrm{W}_{\text {loc }}^{1, p}\left((0,1)^{n-1} ; \mathbb{R}^{m}\right), u \text { 1-periodic in } x_{\alpha}\right\}\right) d t \\
& \geq \int_{0}^{1} \widetilde{W}_{\text {hom }}(t, \bar{F}) d t
\end{aligned}
$$

by Jensen's inequality.

Theorem 5.3 For all $\bar{F} \in \mathbb{M}^{m \times(n-1)}$ we have $W_{0}(\bar{F})=\bar{W}_{\text {hom }}(\bar{F})$.

Proof. With fixed $\eta>0$ let $v$ be a test function for (5.7) such that

$$
\int_{(0,1)^{n}} W_{\mathrm{hom}}\left(x_{n}, D v+\bar{F}\right) d x \leq \bar{W}_{\mathrm{hom}}(\bar{F})+\eta .
$$

By Theorem 4.15 there exists a sequence $v_{j}$ converging to $v$ such that $v_{j}=v$ on $\partial(0,1)^{n}$ (and, hence, in particular $v_{j}$ is 1-periodic in $\left.x_{\alpha}\right)$ and

$$
\int_{(0,1)^{n}} W_{\mathrm{hom}}\left(x_{n}, D v+\bar{F}\right) d x=\lim _{j \rightarrow+\infty} \int_{\Omega_{j} \cap(0,1)^{n}} W\left(D v_{j}+\bar{F}\right) d x .
$$

If we define $u_{j}\left(x_{\alpha}, x_{n}\right)=\frac{1}{j} v_{j}\left(j x_{\alpha}, x_{n}\right)$ then $u_{j} \rightarrow 0$ in $\mathrm{L}^{p}\left((0,1)^{n} ; \mathbb{R}^{m}\right)$ and

$$
\begin{aligned}
\int_{\Omega_{j} \cap(0,1)^{n}} W\left(D v_{j}+\bar{F}\right) d x & =\frac{1}{j^{n-1}} \int_{\Omega_{j} \cap\left((0, j)^{n-1} \times(0,1)\right)} W\left(D v_{j}+\bar{F}\right) d x \\
& =\int_{\Omega_{j^{2}} \cap(0,1)^{n}} W\left(D v_{j}\left(j y_{\alpha}, y_{n}\right)+\bar{F}\right) d y \\
& =\int_{\Omega_{j^{2}} \cap(0,1)^{n}} W\left(D_{\alpha} u_{j}+\bar{F} \mid j D_{n} u_{j}\right) d y \\
& =E_{j}\left(u_{j}+\bar{F} x_{\alpha},(0,1)^{n}\right)
\end{aligned}
$$


hence, we can conclude that

$$
\begin{aligned}
W_{0}(\bar{F}) & \leq \liminf _{j \rightarrow+\infty} E_{j}\left(u_{j}+\bar{F} x_{\alpha},(0,1)^{n}\right) \\
& =\liminf _{j \rightarrow+\infty} \int_{\Omega_{j} \cap(0,1)^{n}} W\left(D v_{j}+\bar{F}\right) d x \\
& \leq \bar{W}_{\text {hom }}(\bar{F})+\eta
\end{aligned}
$$

by (5.6), (5.11), (5.10) and (5.7).

Now we prove the converse inequality. Let $u_{j} \rightarrow 0$ be such that

$$
W_{0}(\bar{F})=\lim _{j \rightarrow+\infty} E_{j}\left(u_{j}+\bar{F} x_{\alpha},(0,1)^{n}\right) .
$$

By [8] Lemma 2.6 we can choose $u_{j} 1$-periodic in $x_{\alpha}$; let $v_{j}$ be defined by $v_{j}(x)=$ $j u_{j}\left(x_{\alpha} / j, x_{n}\right)$. With fixed $j, N \in \mathbb{N},(0,1)^{n}=\bigcup_{m=1}^{N}(0,1)^{n-1} \times((m-1) / N, m / N)$; we can define a function $v_{j, m}$ by setting

$$
v_{j, m}\left(x_{\alpha}, x_{n}\right)= \begin{cases}v_{j}\left(x_{\alpha}, x_{n}+\frac{2 k}{N}\right) & \text { if } \frac{m-1}{N}-\frac{2 k}{N}<x_{n}<\frac{m}{N}-\frac{2 k}{N} \\ v_{j}\left(x_{\alpha}, \frac{2 m}{N}-x_{n}-\frac{2 k+2}{N}\right) & \text { if } \frac{m-1}{N}-\frac{2 k+1}{N}<x_{n}<\frac{m}{N}-\frac{2 k+1}{N}\end{cases}
$$

for $k \in \mathbb{Z}$, which is 1 -periodic in $x_{\alpha}$ and $2 / N$-periodic in $x_{n}$. Hence, we can construct

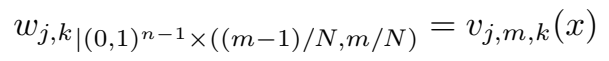

where $v_{j, m, k}(x)=\frac{j}{k} v_{j, m}\left(\frac{k}{j} x\right)$, such that $w_{j, k}$ is $\frac{j}{k}$-periodic in $x_{\alpha}$ and

$$
w_{j, k \mid(0,1)^{n-1} \times((m-1) / N, m / N)} \rightarrow\left(0,\left(\int_{(0,1)^{n}} D_{n} v_{j, m} d x\right) x_{n}\right)=w^{m}
$$

as $k \rightarrow+\infty$, in $\mathrm{L}^{p}\left((0,1)^{n} ; \mathbb{R}^{m}\right)$. In this case the functions $w_{j, k}$ defined as above belong to $\mathrm{W}^{1, p}\left(\Omega_{k} \cap(0,1)^{n} ; \mathbb{R}^{m}\right)$.

Finally, we define $w$ such that

$$
w_{\mid(0,1)^{n-1} \times((m-1) / N, m / N)}=w^{m}
$$

which is 1 -periodic in $x_{\alpha}$. Let

$$
A_{j}^{m / N}=\Omega_{j} \cap\left\{x_{n}=m / N\right\}
$$

and

$$
A_{k}^{m / N}=\Omega_{k} \cap\left\{x_{n}=m / N\right\},
$$

we define

$$
E_{j}^{N}=\bigcup_{m=1}^{N} A_{j}^{m / N} \times((m-1) / N, m / N)
$$


and

$$
E_{k}^{N}=\bigcup_{m=1}^{N} A_{k}^{m / N} \times((m-1) / N, m / N) .
$$

We restrict our analysis to the case where $k / j$ odd, the other case being dealt with by introducing a small error term. Hence, if we use the notation

$$
I_{l}\left(u,(0,1)^{n}\right)=\int_{E_{l}^{N} \cap(0,1)^{n}} W(D u) d x
$$

$(l=j$ or $k)$ we have that

$$
I_{j}\left(v_{j}+\bar{F} x_{\alpha},(0,1)^{n}\right)=I_{k}\left(w_{j, k}+\bar{F} x_{\alpha},(0,1)^{n}\right) .
$$

Reasoning as in Theorems 4.11 and 4.13 we get that

$$
\begin{aligned}
& I_{\mathrm{hom}}\left(w+\bar{F} x_{\alpha},(0,1)^{n}\right)=\Gamma-\lim _{k \rightarrow+\infty} I_{k}\left(w+\bar{F} x_{\alpha},(0,1)^{n}\right) \\
= & \sum_{m=1}^{N} \int_{(0,1)^{n-1} \times((m-1) / N, m / N)} W_{\mathrm{hom}}(m / N, D w+\bar{F}) d x \\
= & \sum_{m=1}^{N} \int_{(0,1)^{n-1} \times((m-1) / N, m / N)} W_{\mathrm{hom}}\left(\frac{\left[x_{n} N\right]+1}{N}, D w+\bar{F}\right) d x \\
= & \int_{(0,1)^{n}} W_{\mathrm{hom}}\left(\frac{\left[x_{n} N\right]+1}{N}, D w+\bar{F}\right) d x \\
\geq & \int_{0}^{1} \widetilde{W}_{\mathrm{hom}}\left(\frac{\left[x_{n} N\right]+1}{N}, \bar{F}\right) d x_{n}
\end{aligned}
$$

by (5.9). Taking the limit as $N \rightarrow+\infty$, we obtain

$$
I_{\text {hom }}\left(w+\bar{F} x_{\alpha},(0,1)^{n}\right) \geq \bar{W}_{\text {hom }}(\bar{F})
$$

by Proposition 5.2. Hence,

$$
\begin{aligned}
E_{j}\left(u_{j}+\bar{F} x_{\alpha},(0,1)^{n}\right) & =\int_{\Omega_{j} \cap(0,1)^{n}} W\left(D v_{j}+\bar{F}\right) d x \\
& \geq \liminf _{k \rightarrow+\infty} I_{k}\left(w_{j, k}+\bar{F} x_{\alpha},(0,1)^{n}\right) \\
& \geq \bar{W}_{\mathrm{hom}}(\bar{F})
\end{aligned}
$$

by (5.11)-(5.13). By the choice of $\left(u_{j}\right)$ we get the desired inequality.

The proof of Theorem 5.1 will be complete once we observe that in the convex case formula (5.5) simplifies that in Theorem 4.13 (see e.g. [6] Section 14.3). 


\subsection{Convergence of minimum problems}

As an application of the $\Gamma$-convergence result of the previous section, we describe the asymptotic behaviour of problems of the form

$$
\begin{aligned}
m_{\varepsilon, \delta}= & \min \left\{\int_{\Omega(\varepsilon, \delta)} W(D u) d x: u \in \mathrm{L}^{p}\left(\omega \times(-\varepsilon, \varepsilon) ; \mathbb{R}^{m}\right),\right. \\
& \left.u_{\mid \Omega(\varepsilon, \delta)} \in \mathrm{W}^{1, p}\left(\Omega(\varepsilon, \delta) ; \mathbb{R}^{m}\right), u=\phi \text { on }(\partial \omega) \times(-\varepsilon, \varepsilon)\right\},
\end{aligned}
$$

where $\phi=\phi\left(x_{\alpha}\right) \in \mathrm{W}^{1, p}\left(\omega ; \mathbb{R}^{m}\right), \Omega(\varepsilon, \delta)$ is given by (1.2) and $f$ and $W$ satisfy the hypotheses of Theorem 5.1. By using Poincaré's inequality it can immediately be checked that problem (5.14) admits at least one solution for each choice of $\varepsilon, \delta>0$. The asymptotic behaviour of these solutions when $\varepsilon \rightarrow 0$ and $\delta<<\varepsilon$ is given by the following result.

Proposition 5.4 Let $\varepsilon$ and $\delta=\delta(\varepsilon)$ satisfy the hypotheses of Theorem 5.1, and for each $\varepsilon$ let $u_{\varepsilon}$ be a solution of (5.14). Then, upon extracting a subsequence, there exist a sequence $\left(v_{\varepsilon}\right)$ in $\mathrm{L}^{p}\left(\omega \times(-1,1) ; \mathbb{R}^{m}\right)$ and a function $w \in \mathrm{W}^{1, p}\left(\omega ; \mathbb{R}^{m}\right)$ such that

(i) $v_{\varepsilon}=u_{\varepsilon}$ on $\Omega(\varepsilon, \delta(\varepsilon))$,

(ii) if $w_{\varepsilon}\left(x_{\alpha}, x_{n}\right)=v_{\varepsilon}\left(x_{\alpha}, \varepsilon x_{n}\right)$, then $w_{\varepsilon}$ converges (with the identification $\left.w(x)=w\left(x_{\alpha}\right)\right)$ to $w$ in $\mathrm{L}^{p}\left(\left(\omega \times(-1,1) ; \mathbb{R}^{m}\right)\right.$,

(iii) $w$ is a solution of the minimum problem

$$
\widetilde{m}_{0}=\min \left\{\int_{\omega} 2 \bar{W}_{\text {hom }}\left(D_{\alpha} u\right) d x_{\alpha}: u \in \mathrm{L}^{p}\left(\omega ; \mathbb{R}^{m}\right), u=\phi \text { on } \partial \omega\right\},
$$

where $\bar{W}_{\text {hom }}$ is defined by (5.4) and (5.5),

(iv) $m_{\varepsilon, \delta(\varepsilon)} / \varepsilon$ converges to $\widetilde{m}_{0}$.

Proof. Note that, in the notation of Theorem 5.1, $\widetilde{u}_{\varepsilon}$ defined by $\widetilde{u}_{\varepsilon}\left(x_{\alpha}, x_{n}\right)=$ $u_{\varepsilon}\left(x_{\alpha}, \varepsilon x_{n}\right)$ is a solution of

$$
\begin{aligned}
\widetilde{m}_{\varepsilon}=\frac{1}{\varepsilon} m_{\varepsilon, \delta(\varepsilon)} & =\min \left\{\int_{\Omega_{\varepsilon}} W\left(D_{\alpha} u, \frac{1}{\varepsilon} D_{n} u\right) d x: u \in \mathrm{L}^{p}\left(\omega \times(-1,1) ; \mathbb{R}^{m}\right)\right. \\
u_{\mid \Omega_{\varepsilon}} & \left.\in \mathrm{W}^{1, p}\left(\Omega_{\varepsilon} ; \mathbb{R}^{m}\right), u=\phi \text { on }(\partial \omega) \times(-1,1)\right\}
\end{aligned}
$$

By [8] Remark 2.3, upon extracting a subsequence, there exist $w_{\varepsilon} \in \mathrm{L}^{p}((\omega \times$ $\left.(-1,1) ; \mathbb{R}^{m}\right)$ converging to some $w$ in $\mathrm{L}^{p}\left(\left(\omega \times(-1,1) ; \mathbb{R}^{m}\right), D_{n} w=0\right.$ and $w_{\varepsilon}=\widetilde{u}_{\varepsilon}$ on $\Omega_{\varepsilon}$. By the well-known property of the convergence of minima and minimizers

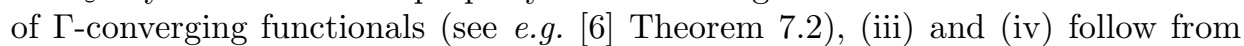
Theorem 5.1, since the $\Gamma$-limit is not influenced by the boundary value $\phi$ (see 8] Lemma 2.6). 
Acknowledgements We gratefully acknowledge stimulating discussions with I. Fonseca, and a very careful reading of the manuscript by the anonymous referee. The research of $\mathrm{AB}$ was partially supported by Marie-Curie fellowship ERBFMBICT972023 of the European Union program "Training and Mobility of Researchers", and benefitted from the hospitality of the Max-Planck Institute for Mathematics in the Sciences, Leipzig (Germany).

\section{References}

[1] G. Anzellotti, S. Baldo and D. Percivale, Dimension-reduction in variational problems, asymptotic development in $\Gamma$-convergence and thin structures in elasticity, Asymptotic Anal. 9 (1994), 61-100.

[2] K. Bhattacharya and A. Braides, Thin films with many small cracks. Preprint SISSA 1999.

[3] K. Bhattacharya and R.D. James, A theory of thin films of martensitic materials with applications to microactuators, J. Mech. Phys. Solids 47 (1999), 531-576.

[4] A. Braides, Homogenization of some almost periodic functional, Rend. Accad. Naz. Sci. XL 103 (1985), 313-322.

[5] A. Braides, $\Gamma$-convergence for Beginners, Oxford University Press, Oxford, to appear.

[6] A. Braides and A. Defranceschi, Homogenization of Multiple Integrals, Oxford University Press, Oxford, 1998.

[7] A. Braides and I. Fonseca, Brittle thin films, Appl. Math. Optim., to appear.

[8] A. Braides, I. Fonseca and G. Francfort, 3D-2D asymptotic analysis for inhomogeneous thin films, to appear.

[9] R. Brizzi and J.P. Chalot, Boundary homogenization and Neumann boundary value problems, Ric. Mat. 46 (1997), 341-387.

[10] G. Buttazzo, Semicontinuity, Relaxation and Integral Representation in the Calculus of Variations, Longman, Harlow, 1989.

[11] D. Caillerie, Thin elastic and periodic plates, Math. Meth Appl. Sci. 6 (1984), 159-191.

[12] C. Castaign and M. Valadier, Convex Analysis and Measurable Multifunctions, Springer Verlag, Berlin, 1977.

[13] G. Dal Maso, An Introduction to Г-convergence, Birkhäuser, Boston, 1993. 
[14] E. De Giorgi and T. Franzoni, Su un tipo di convergenza variazionale, Atti Accad. Naz. Lincei Rend. Cl. Sci. Mat. Fis. Natur. 58 (1975), 842-850.

[15] L.C. Evans and R.F. Gariepy, Measure Theory and Fine Properties of Functions, CRC Press, Ann Harbor, 1992.

[16] I. Fonseca and G. Francfort, 3D-2D asymptotic analysis of an optimal design problem for thin films, J. reine angew. Math. 505 (1998), 173-202.

[17] I. Fonseca, S. Müller and P. Pedregal, Analysis of concentration and oscillation effects generated by gradients. SIAM J. Math. Anal. 29 (1998), 736-756.

[18] G.A. Francfort and S. Müller, Combined effects of homogenization and singular perturbations in elasticity. J. reine angew. Math. 454 (1994), 1-35.

[19] H. Le Dret and A. Raoult, The nonlinear membrane model as variational limit of nonlinear three-dimensional elasticity, J. Math. Pures Appl. 74 (1995), $549-578$.

[20] R.V. Kohn and M. Vogelius, A new model for thin plates with rapidly varying thickness. II: a convergence proof, Quarterly Appl. Math. 43 (1985), 1-22.

[21] P. Marcellini, Periodic solutions and homogenization of nonlinear variational problems, Ann. Mat. Pura Appl. 117 (1978), 481-498.

[22] S. Müller, Homogenization of nonconvex integral functionals and cellular elastic materials, Arch. Rational Mech. Anal. 99 (1987), 189-212.

[23] Y.C. Shu, Heterogeneous thin films of martensitic materials, Preprint, 1998. 